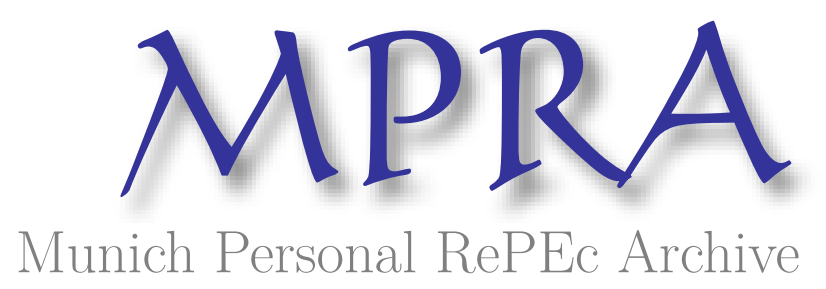

\title{
Spatial Discounting, Fourier, and Racetrack Economy: A Recipe for the Analysis of Spatial Agglomeration Models
}

Akamatsu, Takashi and Takayama, Yuki and Ikeda, Kiyohiro

18 August 2009

Online at https://mpra.ub.uni-muenchen.de/21738/

MPRA Paper No. 21738, posted 31 Mar 2010 05:45 UTC 


\title{
Spatial Discounting, Fourier, and Racetrack Economy: A Recipe for the Analysis of Spatial Agglomeration Models
}

\author{
By Takashi Akamatsu, Yuki Takayama and Kiyohiro Ikeda
}

\begin{abstract}
We provide an analytical approach that facilitates understanding the bifurcation mechanism of a wide class of economic models involving spatial agglomeration of economic activities. The proposed method overcomes the limitations of the Turing (1952) approach that has been used to analyze the emergence of agglomeration in the multi-regional core-periphery (CP) model of Krugman (1993, 1996). In other words, the proposed method allows us to examine whether agglomeration of mobile factors emerges from a uniform distribution and to analytically trace the evolution of spatial agglomeration patterns (i.e., bifurcations from various polycentric patterns as well as a uniform pattern) that these models exhibit when the values of some structural parameters change steadily. Applying the proposed method to the multi-regional $\mathrm{CP}$ model, we uncover a number of previously unknown properties of the $\mathrm{CP}$ model, and notably, the occurrence of "spatial period doubling bifurcation" in the CP model is proved.
\end{abstract}

Keywords: economic geography, agglomeration, stability, bifurcation, gravity laws JEL classification: R12, R13, F12, F15, F22, C62, C65

\section{Introduction}

Most of the world's population and economic activities are strikingly concentrated in a limited number of areas. Although thousands of articles have been devoted to explain the prevalence of this pattern, the core-periphery (CP) model developed by Krugman (1991) is the first successful attempt to explain this in a full-fledged general equilibrium framework (Fujita and Thisse (2009)). The CP model introduces the Dixit-Stiglitz (1977) model of monopolistic competition and increasing returns into spatial economics and provides a basic framework by which to describe how the interaction among increasing returns, transportation cost, and factor mobility can cause the formation of economic agglomeration in geographical space. These new modeling techniques further led to the development of a new branch of spatial economics, known as "new economic geography (NEG)", which has become a fastgrowing field. Over the past few decades, numerous extensions of the original CP model have appeared ${ }^{1}$, and notably, there has been a proliferation of theoretical and empirical research applying the NEG theory framework to deal with various policy

\footnotetext{
${ }^{1}$ For comprehensive reviews of the literature on the NEG/CP models, see the following monographs and handbooks: Fujita et al. (1999), Papageorgiou and Pines (1999), Fujita and Thisse (2002), Baldwin et al. (2003), Henderson and Thisse (2004), and Combes et al. (2008), and Glaeser (2008).
} 
issues, such as trade policy, taxation, or regional redistribution in recent years (Baldwin et al. (2003), Combes et al. (2009)).

As indicated by its remarkable expansion, the NEG theory has the potential to provide a solid basis for predicting and evaluating the long-run effects of various economic policy proposals. There remain, however, some fundamental limitations that must be addressed before the NEG theory can be confirmed to provide a sound foundation for such applications. One of the most relevant issues is that the theoretical results are mainly restricted to two-region cases. Even though some efforts to overcome this limitation have been made in the early stages of development of the NEG (e.g., Krugman (1993, 1996) and Fujita et al. (1999)), theoretical studies on the NEG models have focused almost exclusively on two regions in the last decade.

Although clearly the correct starting point for analysis, two-region models have several limitations. First, these models can neither describe nor explain a rich variety of polycentric patterns that are prevalent in real-world economies. Second, these models cannot provide an adequate framework to consider spatial interactions in a well-defined sense. For example, consider the concept of market accessibility, the differences among regions of which, as evidenced by numerous empirical studies, are major factors that lead to various forms of spatial agglomeration. Note that this concept is defined as the relative position of each region within the entire network of interactions. Due to the existence of such network effects, the impacts of a change in market accessibility in a multi-regional system are quite different from those in a tworegion system, the topology of which is too simple to capture the network effects (Behrens et al. (2007)).

In view of the limitations of two-region models, it remains unclear as to whether we can extrapolate the predictions and implications derived from two-regional analysis to a multi-regional system (Behrens and Thisse (2007)). Therefore, it is fair to say that "real-world geographical issues cannot be easily mapped into tworegional analysis"_(Fujita et al. (1999), pp.79), and the multi-regional framework is a prerequisite for systematic empirical research as well as for practical applications of the NEG theory.

In the present paper, an attempt is made to clarify the spatial agglomeration properties of the multi-regional CP model. As described in the NEG literature, the two-region CP model exhibits a bifurcation from a symmetric equilibrium (spatially uniform distribution of population) to an asymmetric equilibrium (agglomeration of population), depending on the values of certain structural parameters. In analyzing the multi-regional CP model, the most important, yet most difficult, step is examining the 
more complex bifurcation (i.e., evolution of spatial agglomeration structure). In the present paper, we investigate the mechanism of the bifurcation through a novel analytical approach that we shall explain later in this section. For the analysis, we first present a multi-regional version of Pfluger's (2004) two-regional CP model, which is a slight modification of Krugman's original CP model, so that short-run equilibrium is obtained analytically ${ }^{2}$. We then show a complete picture of the evolutionary processes of agglomeration patterns in the CP model with $K$ regions equidistantly located on a circumference. The term evolutionary process means that we consider a process in which transportation cost parameter $\tau$ steadily decreases over time, starting from a very high value of $\tau$, at which a uniform distribution of firms/labors is a stable equilibrium.

It is first shown that the critical value of $\tau$ at which the agglomeration structure first emerges (i.e., a first bifurcation occurs) and the associated agglomeration pattern can be obtained analytically. While the first bifurcation is the final and only bifurcation in conventional two-region models, the multi-regional CP model does not end the story. It is indeed proved that the spatial configuration of population evolves over time with further decreases in $\tau$, accompanied by a second bifurcation. The analysis of these two bifurcations further reveals that they share a common property, namely, the number of core regions (in which firms/workers agglomerate) is reduced by half and the spacing between each pair of neighboring core-regions (agglomeration shadow) doubles after each bifurcation. This suggests a general rule whereby the multi-regional CP economy exhibits the spatial period doubling bifurcation (SPDB). Finally, a proof of this conjecture, including a general analytical formula to predict the occurrence of the SPDB, is presented ${ }^{3}$. In addition to these results, we further uncover the effect of the heterogeneity of consumers on agglomeration patterns. Specifically, it is deduced that the CP model may exhibit repeated agglomeration and dispersion with the steady decrease in $\tau$, which provides a generalization, as well as a theoretical explanation, of several bell-shaped development results reported in studies on two-region CP models ${ }^{4}$.

While the present paper elucidates the intrinsic properties of the multi-regional CP model, another unique contribution is that an analytical approach that facilitates

\footnotetext{
${ }^{2}$ Although the approach presented herein can be applied to Krugman's (1991) original CP model, we chose this model for clarity of exposition. Essentially, the same results as those obtained for the present model are obtained for variants of the Krugman's model (e.g., Forslid and Ottaviano (2003), Ottaviano et al. (2002)), as well as the original Krugman's model.

${ }^{3}$ While Krugman $(1993,1996)$ has presented some numerical results that can be regarded as special examples of the SPDB, no study has presented a rigorous proof of the general SPDB in the CP model.

${ }^{4}$ See Tabuchi (1998), Helpman (1998), and Murata (2003).
} 
understanding bifurcation properties of a wide class of economic models (as well as the CP models) involving spatial agglomeration of economic activities is provided. The approach presented in the present paper is characterized by the following three tools: (A) spatial discounting matrix (SDM), (B) discrete Fourier transformation (DFT), and (C) a system of regions on a circumference (racetrack economy (RE)). We outline our approach by briefly reviewing each of these tools/concepts in turn.

(A) Geographical location patterns of economic activities are largely shaped by the tension between agglomeration forces and dispersion forces. Take the CP model with two regions, for example. Suppose that a firm moves from one region to another region (say, region 1). This generates an agglomeration force through a circular causation of forward linkages (market access effect on consumers) and backward linkages (market access effect on firms). The former is the incentive for workers to be close to the producers of consumer goods, and the latter is the incentive for producers to concentrate at locations where the market is greater. In opposition to the agglomeration force, a dispersion force (market crowding effect) is also at work. The increased competition in region 1 gives other firms in this region the incentive to move to other regions. If the agglomeration force is strong enough to overcome the dispersion force, then the core-periphery location pattern emerges in the two-regional economy.

In a multi-regional economy with more than two regions, it should be emphasized that these agglomeration/dispersion forces not only affect region 1 but also spill over to every other region with a different strength due to the existence of transportation costs and imperfect competition of markets. Furthermore, in the multi-regional CP model, the manner in which each of the agglomeration/dispersion forces decays with distance (i.e., these forces are spatially discounted) is described by gravity laws ${ }^{5}$. Accordingly, the spatial extent and patterns of such spillover effects governed by the gravity laws determine the geographical location patterns of firms and laborers.

A key component of the multi-regional CP model for systematically dealing with

\footnotetext{
${ }^{5}$ The concepts of spatial discounting, accessibility, and gravity laws have a long tradition in fields dealing with spatial aspects of economic activities. Examples include geography, transportation science, location theory, urban economics, regional economics, and international economics. Early empirical studies of gravity laws in the social science literature extend back to Ravenstein (1885), Young (1924), Reilly (1931), Zipf (1946), Voorhees (1955), and Tinbergen (1962). It has been well established in regional science and transportation science that gravity laws can be theoretically derived from the maximum entropy principle (e.g., Wilson (1967, 1970), Smith (1976), Anas (1983), and Smith and Sen (1995)) or random utility (discrete choice) theory (e.g., Anderson et al. (1992)). Since Anderson (1979), it has become increasingly apparent that the gravity models can also be derived from several different models with micro economic underpinning (e.g., Bergstrand (1989), Evenett and Keller (2002), and Anderson and Wincoop (2003).
} 
the spatial patterns of the spillover effect is the spatial discounting matrix (SDM), the elements of which represent the spatial discounting factors (SDF) for all pairs of regions. (For the definitions of SDF and SDM, see Section 3.) The SDM encapsulates the essential information required to analyze the multi-regional CP model. In order to determine what types of spatial concentration-dispersion patterns can emerge in the $\mathrm{CP}$ model for a racetrack economy, it suffices $a$ ) to obtain the eigenvalues of the SDM, and $b$ ) to represent the Jacobian matrix of the indirect utility as a function of the SDM, as shown later herein. Note that, although this fact holds for a wide variety of models dealing with the formation of spatial patterns in economic activities, we herein restrict ourselves to demonstrating this fact using the $\mathrm{CP}$ model.

(B) Discrete Fourier transformation (DFT) is a simple and powerful technique for obtaining the essential indices (eigenvalues and eigenvectors) by which to predict the emergence of agglomeration. This may at first appear to be simply a computational issue, but its significance in the analysis of the CP model deserves further explanation.

Most economic models involving spatial agglomeration, including the CP model, usually predict the existence of multiple equilibria. As a manner of refining the set of equilibria, it is customary in the NEG literature to assess the stability of equilibria by assuming a particular class of adjustment process (e.g., replicator dynamics) used in evolutionary game theory ${ }^{6}$. As demonstrated by Papageorgiou and Smith (1983), Krugman (1996), and Fujita et al. (1999), combining the evolutionary approach with Turing's approach (Turing, 1952) provides a very effective method of investigating the emergence of agglomeration. In this approach, the investigation is concerned primarily with the onset of the instability of a uniform equilibrium distribution of mobile workers (which implies the emergence of some agglomeration). The procedure for examining the instability is based on the eigenvalues $\mathbf{g}=\left\{g_{k}, k=1,2, \ldots, K\right\}$ of the Jacobian matrix of the adjustment process evaluated at the symmetric equilibrium (flat earth equilibrium). Accordingly, if the eigenvalues $\mathbf{g}$ are represented as functions of the key parameters of the $\mathrm{CP}$ model (e.g., transport cost parameter $\tau$ ), we can predict whether a particular agglomeration pattern (bifurcation) will emerge with changes in the parameter values.

While Turing's approach offers a remarkable method for assessing the emergence of agglomeration, it has two important limitations. First, Turing's approach deals with only the first stage of agglomeration, in which the value of transportation cost steadily decreases, and cannot give a general description of what happens thereafter. Second,

\footnotetext{
${ }^{6}$ In the present paper, we use perturbed best response dynamics (e.g., Fudenberg and Levine (1998), Vega-Redondo (2003), Sandholm (2009)), instead of replicator dynamics, because the former is suitable for dealing with the CP model with heterogeneous agents. (See Section 4.)
} 
despite its conceptual simplicity, performing the eigenvalue analysis in a straightforward manner becomes very complicated, even for a model with only a few cities, and it is, in general, almost impossible to analytically obtain the eigenvalues $\mathbf{g}$ evaluated for an arbitrary configuration of mobile workers. This is one of the most difficult obstacles to clarifying the general properties of the multi-regional CP model.

As shown in the present paper, however, the DFT coupled with the racetrack economy resolves these limitations. The eigenvalues $\mathbf{g}$ of the Jacobian of the CP model in the racetrack economy can be represented as a simple unimodal function of the eigenvalues of either the SDM or its submatrices. More precisely, the $k$ th eigenvalue $g_{k}$ for the flat earth equilibrium reduces to a quadratic function of the $k$ th eigenvalue $f_{k}$ of the SDM (see Section 5), whereas for the equilibrium configurations that emerge at later stages during the course of steadily decreasing transportation cost, a simple technique allows us to obtain each of the eigenvalues $\mathbf{g}$ as a function of the eigenvalues of the submatrices of the SDM (see Section 6). This means that, if the eigenvalues of the SDM and its submatrices are obtained analytically, we can completely characterize the intrinsic nature of the $\mathrm{CP}$ model without resorting to numerical simulations. The DFT offers a powerful means for obtaining the very eigenvalues, especially when combined with the racetrack economy explained below.

(C) The racetrack economy (RE) is an idealized spatial setting in which locations (regions or cities) are spread equidistantly over the circumference of a circle. Perhaps, due to its apparently unrealistic setting, the significance of this spatial setting appears to have been neither fully recognized nor exploited in previous studies on the NEG models following the studies of Krugman (1996) and Fujita et al. (1999) ${ }^{7}$. In fact, few studies have used this approach to analyze the multi-regional CP model. In our view, however, the analysis of the racetrack economy is, for several reasons, indispensable in fully clarifying the intrinsic self-organizing nature of a wide variety of models dealing with the spatial agglomeration of economic activities.

First, the RE is helpful in obtaining a systematic overview of these spatial agglomeration models. Note that these modes have not been systematically compared in a unified spatial setting, despite the fact that the resulting location patterns are significantly affected by spatial boundary conditions or location specific conditions. In the RE, these effects are completely removed, so that we can isolate the selforganizing properties in a pure form. Accordingly, the RE provides a benchmark circumstance or an ideal test-bed designed to compare the intrinsic properties of these

\footnotetext{
${ }^{7}$ Before Krugman's research $(1993,1996)$, there were few studies using a circular city model (e.g., Salop (1979), Novshek (1980), Papageorgiou and Smith (1983), and Eaton and Wooders (1985)).
} 
models, and such comparisons should facilitate the refinement of a body of knowledge on these models into a coherent and systematic theory.

Second, the RE with discrete locations greatly facilitates the eigenvalue analysis of these spatial agglomeration models. More specifically, the SDM for the RE reduces to a circulant matrix, which allows us to obtain the analytical expression of the eigenvalues of the SDM through a simple matrix operation, namely, the DFT of the first row vector of the $\mathrm{SDM}^{8}$. Furthermore, each row vector of the DFT matrix (i.e., each eigenvector) represents the agglomeration patterns of firms/workers by the configuration pattern of the entries (see Section 4). Accordingly, the eigenvectors and the associated eigenvalues $\mathbf{g}$ thus obtained provide a simple method by which to predict what types of agglomeration patterns emerge for an arbitrary given condition.

Finally, note that the results obtained for the RE have a significant degree of generality. From a mathematical point of view, the essence of the RE lied in its periodic boundary condition, which yields structural symmetry of the system. The bifurcation properties of such systems (with symmetric structure) often have some general regularities described by the group-theoretic bifurcation theory (e.g., Golubitsky and Schaeffer (1985)). As can be inferred from this theoretical link, most of the intrinsic properties of the CP models obtained in the RE are not restricted to a one-dimensional space, but rather hold for a two-dimensional space with a periodic boundary. Therefore, the approach presented in the present paper can be easily extended to a two-dimensional space, although the present paper considers only the one-dimensional case.

Thus, by laying out these three tools/concepts (A), (B), and (C), the present study makes a unique contribution to the understanding of the bifurcation mechanism of a variety of economic agglomeration models, as well as the $\mathrm{CP}$ model. Although each of these tools/concepts is rather well established in the literature, the present study is unique in the sense that 1) it sheds new light on the essential role of the spatial discounting factor (SDF) in the context of the bifurcation mechanism/analysis of the economic models involving spatial agglomeration, 2) it provides an analytically tractable method that allows the evolutionary processes of spatial agglomeration, as well as the emergence of agglomeration, to be traced by effectively combining discrete Fourier transformation (DFT) with the circulant properties of the spatial

\footnotetext{
${ }^{8}$ The use of DFT combined with a circulant matrix is not an esoteric technique. Rather, this is an indispensable technique in a wide variety of fields that deal with spatial patterns, including time-series analysis in econometrics, macro economics, and finance; signal processing, image processing, and pattern recognition in computer science; morphogenesis in theoretical biology; and phase transitions in materials in statistical physics. Therefore, it is natural that economic models involving spatial patterns of agglomeration might be analyzed using these tools.
} 
discounting matrix (SDM) in the discrete racetrack economy (RE), and 3) it demonstrates the usefulness of the proposed method by uncovering a number of previously unknown properties of the CP model.

The remainder of the present paper is organized as follows. Section 2 discusses related research. Section 3 formulates a multi-regional CP model and defines the stability of the equilibrium of the model. Section 4 defines the SDM in a racetrack economy, the eigenvalues of which are provided by DFT. In Section 5, several types of bifurcations from a uniform distribution are analyzed, which also serves as a preliminary step for the analysis of the SPDB in Section 6. Conclusions are presented in Section 7.

\section{Related Research}

Since the seminal work of Krugman (1991), an enormous number of studies on the CP model and its variants have been published. However, since the work of Krugman (1993, 1996) and Fujita et al. (1999), theoretical studies on the CP/NEG models have been almost exclusively limited to two-region cases. A few exceptions have dealt with CP models having more than two regions (Mossay (2003), Tabuchi et al. (2005), Picard and Tabuchi (2009), Oyama (2009), Akamatsu and Takayama (2009a), Tabuchi and Thisse (2009), and Ikeda et al. (2009)).

Krugman (1993) presented a few numerical simulation runs for the CP model in which 12 regions are equidistantly located on a circumference. The resultant locations of population, in which three types of evenly spaced agglomeration patterns (monocentric, duo-centric, and tri-centric patterns) emerge, suggests a certain similarity to Christaller's central-place patterns (Christaller, 1933). The numerical experiments, however, are limited to a particular set of parameter values, and no theoretical analysis is given. Krugman (1996, Appendix) and Fujita et al. (1999, Chapter 6) analyzed the $\mathrm{CP}$ model with a continuum of locations on a circumference. Using Turing's approach (Turing, 1952), they succeeded in showing the conditions for the instability of the flat earth equilibrium. Their analysis, however, provides only the initial period of agglomeration (a first bifurcation) when the transportation cost steadily increases. In order to analyze the possible bifurcations in the later stages, they resort to rather ad hoc numerical simulations. Thus, it is fair to say that their investigations do not systematically show the general properties of the multi-regional CP model, although these studies do provide valuable insight.

Recently, a few studies have carefully re-examined the robustness of Krugman's 
findings in the CP model with a continuous-space racetrack economy. Mossay (2003) theoretically qualified Krugman's results in the case of workers' heterogeneous preferences for location. More recently, Picard and Tabuchi (2009) examined the impact of the shape of transport costs on the structure of spatial equilibria. These studies, however, focus only on the first stage of agglomeration as the local instability of a uniform equilibrium distribution. Therefore, the same limitation as Krugman's approach applies to these studies. Tabuchi et al. (2005) studied the impact of decreasing transport costs on the size and number of cities in a multi-regional model that extends a two-regional CP model by Ottaviano et al. (2002). Oyama (2009) showed that the multi-regional $\mathrm{CP}$ model admits a potential function, which allowed to identify a stationary state that is uniquely absorbing and globally accessible under the perfect foresight dynamics. However, these analyses are restricted to a very special class of transport geometry in which regions are pairwise equidistant. Akamatsu and Takayama (2009a) analyzed a pair of multi-regional models that extends the two-region CP models developed by Forslid and Ottaviano (2003) and Pflüger (2004). Although they demonstrated the evolutionary process of spatial agglomeration in these models, their analysis is only restricted to the case of fourregion models.

Only Tabuchi and Thisse (2009) and Ikeda et al. (2009) have discussed the general regularities of bifurcations in the multi-regional $\mathrm{CP}$ model. In the former study, which was conducted independently at approximately the same time as the latter study, Tabuchi and Thisse predicted that the CP model could exhibit a spatial perioddoubling bifurcation (SPDB) pattern. They also showed (by a numerical example) that a hierarchical urban system structure emerges for the $\mathrm{CP}$ model with multiple industries. Although some of their results on the bifurcation properties of the $\mathrm{CP}$ model share certain similarity with our findings, the purpose and the emphasis of their study was significantly different from ours. They attempted to derive an urban hierarchical principle from the CP model. Moreover, unlike our study, they appeared not to recognize the essential role of the SDM in the bifurcation analysis and did not provide a general methodology that is applicable to the analysis of a wider class of models other than the CP model, Accordingly, their analysis is specific to the CP model, in which no simple relations between the Jacobian matrix and the SDF are shown.

In the latter study, Ikeda et al. presented an approach to understanding the bifurcation behaviors of the multi-regional CP model, combining the group-theoretic bifurcation theory with the computational bifurcation theory. They also predicted the 
possibility of the SPDB based on the former theory and then demonstrated its existence using numerical examples based on the latter theory. Admittedly, the former theory is rather abstract and cannot provide a concrete prediction (e.g., the former theory cannot answer with certainty whether the CP model exhibits the SPDB without eigenvalue analysis), although the former theory provides a very general framework for bifurcation analysis. In contrast, the latter theory allows us to obtain very concrete (numerical) predictions, although the range of predictions is limited to certain specific cases. Thus, the present paper bridges the gap between these two theories, by providing a methodology that allows us to analytically predict the general bifurcation properties of a wide class of economic models involving spatial agglomeration of economic activities.

\section{The Core-Periphery Model}

In this section, we present the multi-regional $\mathrm{CP}$ model. Although the basic assumptions of this model are the same as those of Pflüger (2004) ${ }^{9}$, except for the number of regions, for the sake of completeness, we present the basic assumptions of this model in Section 3.1. The short-run equilibrium is described in Section 3.2. The long-run equilibrium and its stability are defined in Section 3.3.

\subsection{Basic Assumptions}

The economy is composed of $K$ regions (labeled $i=0,1, \ldots, K-1$ ), two factors of production (skilled and unskilled labor), and two sectors (agriculture $A$ and manufacturing $\mathrm{M}$ ). There are $H$ skilled and $L=K$ unskilled workers, who consume two final goods, namely, horizontally differentiated M-sector goods and homogeneous A-sector goods, and supply one unit of his type of labor inelastically. Skilled workers are mobile across regions. The number of skilled workers in region $i$ is denoted by $h_{i}$. Unskilled workers are immobile and equally distributed across all regions (i.e., the number of unskilled workers in each region is one).

Preferences $U$ over the M- and A-sector goods are identical across individuals. The utility of an individual in region $i$ is given by

$$
U\left(C_{i}^{M}, C_{i}^{A}\right)=\mu \ln C_{i}^{M}+C_{i}^{A},
$$

where $\mu>0$ is the constant expenditure share on industrial varieties, $C_{i}^{A}$ is the

\footnotetext{
${ }^{9}$ The basic assumptions of this model are the same as those of Krugman (1991), except for the functional forms of a utility function and a production function. Pflüger (2004) replaces the utility function of Krugman with that of the international trade model of Martin and Rogers (1995) and the production function of Krugman with that of Flam and Helpman (1987).
} 
consumption of the A-sector product in region $i$, and $C_{i}^{M}$ is the manufacturing aggregate in region $i$ and is defined as

$$
C_{i}^{M} \equiv\left(\sum_{j} \int_{k \in n_{j}} q_{j i}(k)^{(\sigma-1) / \sigma} d k\right)^{\sigma /(\sigma-1)},
$$

where $q_{j i}(k)$ is the consumption in region $i$ of a variety $k \in\left[0, n_{j}\right]$ produced in region $j, n_{j}$ is the number of varieties produced in region $j$, and $\sigma>1$ is the constant elasticity of substitution between any two varieties. The budget constraint is given by

$$
C_{i}^{A}+\sum_{j} \int_{k \in n_{j}} p_{j i}(k) q_{j i}(k) d k=Y_{i}
$$

where $p_{j i}(k)$ is the price of a variety $k$ in region $i$ produced in region $j$ and $Y_{i}$ is the income of an individual in region $i$.

An individual in region $i$ maximizes (3.1) subject to (3.2). This yields the following demand functions:

$$
\begin{aligned}
& C_{i}^{A}=Y_{i}-\mu, C_{i}^{M}=\mu / \rho_{i}, \\
& q_{j i}(k)=\mu\left\{p_{j i}(k)\right\}^{-\sigma} / \rho_{i}^{1-\sigma},
\end{aligned}
$$

where $\rho_{i}$ denotes the price index of the differentiated product in region $i$, which is given by

$$
\rho_{i}=\left(\sum_{j} \int_{k \in n_{j}} p_{j i}(k)^{1-\sigma} d k\right)^{1 /(1-\sigma)} .
$$

Since the population in region $i$ is $h_{i}+1$, we have the total demand $Q_{j i}(k)$ in region $i$ for a variety $k$ produced in region $j$ :

$$
Q_{j i}(k)=\frac{\mu\left\{p_{j i}(k)\right\}^{-\sigma}}{\rho_{i}^{1-\sigma}}\left(h_{i}+1\right) .
$$

The A-sector is perfectly competitive and produces homogeneous goods under constant returns to scale technology, which requires one unit of unskilled labor in order to produce one unit of output. For simplicity, we assume that the A-sector goods are transported freely between regions and are chosen as the numéraire. These assumptions mean that, in equilibrium, the wage of low-skilled worker $w_{i}^{L}$ is equal to that in all regions (i.e., $w_{i}^{L}=1 \quad \forall i$ ).

The M-sector output is produced under increasing returns to scale and Dixit-Stiglitz monopolistic competition. A firm incurs a fixed input requirement of $\alpha$ units of skilled 
labor and a marginal input requirement of $\beta$ units of unskilled labor. Given the fixed input requirement $\alpha$, the skilled labor market clearing implies that, in equilibrium, the number of firms in region $i$ is determined by $n_{i}=h_{i} / \alpha$. An M-sector firm located in region $i$ maximizes profit:

$$
\Pi_{i}(k)=\sum_{j} p_{i j}(k) Q_{i j}(k)-\left(\alpha w_{i}+\beta x_{i}(k)\right),
$$

where $w_{i}$ is the wage of the skilled worker and $x_{i}(k)$ is the total supply. The transportation costs for $\mathrm{M}$-sector goods are assumed to take on iceberg form. That is, for each unit of the M-sector goods transported from region $i$ to region $j \neq i$, only a fraction $1 / \phi_{i j}<1$ arrives. Thus, the total supply $x_{i}(k)$ is given by

$$
x_{i}(k)=\sum_{j} \phi_{i j} Q_{i j}(k)
$$

Since we have a continuum of firms, each firm is negligible in the sense that its action has no impact on the market (i.e., the price indices). Hence, the first-order condition for profit maximization gives

$$
p_{i j}(k)=\frac{\sigma \beta}{\sigma-1} \phi_{i j} .
$$

This expression implies that the price of the M-sector product does not depend on variety $k$, so that $Q_{i j}(k)$ and $x_{i}(k)$ do not depend on $k$. Thus, we describe these variables without argument $k$. Substituting (3.7) into (3.4), the price index becomes

$$
\rho_{i}=\frac{\sigma \beta}{\sigma-1}\left(\sum_{j} h_{j} d_{j i}\right)^{1 /(1-\sigma)},
$$

where $d_{j i} \equiv \phi_{j i}^{1-\sigma}$ is a spatial discounting factor between regions $j$ and $i$ from (3.5) and (3.8), $d_{j i}$ is obtained as $\left(p_{j i} Q_{j i}\right) /\left(p_{i i} Q_{i i}\right)$, which means that $d_{j i}$ is the ratio of total expenditure in region $i$ for each $\mathrm{M}$-sector product produced in region $j$ to the expenditure for a domestic product.

\subsection{Short-run Equilibrium}

In the short run, skilled workers are immobile between regions, that is, their spatial distribution $\left(\mathbf{h} \equiv\left[h_{0}, h_{1}, \ldots, h_{K-1}\right]^{\mathrm{T}}\right)$ is assumed to be given. The short-run equilibrium conditions consist of the M-sector goods market clearing condition and the zero-profit condition due to the free entry and exit of firms. The former condition can be written as (3.6). The latter condition requires that the operating profit of a firm is entirely absorbed by the wage bill of its skilled workers: 


$$
w_{i}(\mathbf{h})=\alpha^{-1}\left[\sum_{j} p_{i j} Q_{i j}(\mathbf{h})-\beta x_{i}(\mathbf{h})\right] .
$$

Substituting (3.5), (3.6), (3.7), and (3.8) into (3.9), we have the short-run equilibrium wage:

$$
\begin{aligned}
& w_{i}(\mathbf{h})=\mu \sigma^{-1}\left\{w_{i}^{(H)}(\mathbf{h})+w_{i}^{(L)}(\mathbf{h})\right\}, \\
& w_{i}^{(H)}(\mathbf{h}) \equiv \sum_{j} \frac{d_{i j}}{\Delta_{j}(\mathbf{h})} h_{j}, \quad w_{i}^{(L)}(\mathbf{h}) \equiv \sum_{j} \frac{d_{i j}}{\Delta_{j}(\mathbf{h})},
\end{aligned}
$$

where $\Delta_{j}(\mathbf{h}) \equiv \sum_{k} d_{k j} h_{k}$ denotes the market size of the M-sector in region $j$. Thus, $d_{i j} / \Delta_{j}(\mathbf{h})$ defines the market share in region $j$ of each M-sector product produced in region $i$.

The indirect utility $v_{i}(\mathbf{h})$ is obtained by substituting (3.3b), (3.8), and (3.10) into $(3.1)^{10}$ :

$$
v_{i}(\mathbf{h})=S_{i}(\mathbf{h})+\sigma^{-1}\left\{w_{i}^{(H)}(\mathbf{h})+w_{i}^{(L)}(\mathbf{h})\right\},
$$

where $S_{i}(\mathbf{h}) \equiv(\sigma-1)^{-1} \ln \Delta_{i}(\mathbf{h})$. For the convenience of the analysis in following sections, we express the indirect utility function $\mathbf{v}(\mathbf{h})$ in matrix form by using the spatial discounting matrix $\mathbf{D}$, entry $(i, j)$ of which is $d_{i j}$ :

$$
\mathbf{v}(\mathbf{h})=\mathbf{S}(\mathbf{h})+\sigma^{-1}\left\{\mathbf{w}^{(H)}(\mathbf{h})+\mathbf{w}^{(L)}(\mathbf{h})\right\},
$$

where $\mathbf{S}(\mathbf{h}) \equiv\left[S_{0}(\mathbf{h}), S_{1}(\mathbf{h}), \ldots, S_{K-1}(\mathbf{h})\right]^{\mathrm{T}}$, and $\mathbf{w}^{(H)}$ and $\mathbf{w}^{(L)}$ are defined as

$$
\begin{array}{ll}
\mathbf{w}^{(H)} \equiv \mathbf{M h}, & \mathbf{w}^{(L)} \equiv \mathbf{M} \mathbf{1}, \\
\mathbf{M} \equiv \mathbf{D} \boldsymbol{\Delta}^{-1}, \quad \boldsymbol{\Delta} \equiv \operatorname{diag}\left[\mathbf{D}^{\mathrm{T}} \mathbf{h}\right], \quad \mathbf{1} \equiv[1,1, \ldots, 1]^{\mathrm{T}} .
\end{array}
$$

\subsection{Adjustment Process, Long-run Equilibrium and Stability}

In the long run, the skilled workers are inter-regionally mobile. They are assumed to be heterogeneous in their preferences for location choice. That is, the indirect utility for an individual $s$ in region $i$ is expressed as

$$
v_{i}^{(s)}(\mathbf{h})=v_{i}(\mathbf{h})+\varepsilon_{i}^{(s)},
$$

where $\varepsilon_{i}^{(s)}$ denotes the utility representing the idiosyncratic taste for residential location, and $\left\{\varepsilon_{i}^{(s)}, \forall s\right\}$ is continuously distributed across individuals.

We present the dynamics of the migration of the skilled workers in order to define

\footnotetext{
${ }^{10}$ We ignore the constant terms and the coefficient $\mu$, which have no influence on the results in the following sections.
} 
the long-run equilibrium and its stability with respect to small perturbations (i.e., local stability). We assume that at each time period $t$, the opportunity for skilled workers to migrate emerges according to an independent Poisson process with arrival rate $\lambda$. That is, for each time interval $[t, t+d t)$, a fraction $\lambda d t$ of skilled workers have the opportunity to migrate. Given an opportunity at time $t$, each worker chooses the region that provides the highest indirect utility $v_{i}^{(s)}(\mathbf{h}(t))$, which depends on the current distribution $\mathbf{h}(t) \equiv\left[h_{0}(t), h_{1}(t), \ldots, h_{K-1}(t)\right]^{\mathrm{T}}$. The fraction $P_{i}(\mathbf{h}(t))$ of skilled workers who choose region $i$ at time $t$ is given by $P_{i}(\mathbf{h}(t))=\operatorname{Pr}\left[v_{i}^{(s)}(\mathbf{h}(t))>v_{j}^{(s)}(\mathbf{h}(t)) \forall j \neq i\right]$. Thus, we have

$$
h_{i}(t+d t)=(1-\lambda d t) h_{i}(t)+\lambda d t H P_{i}(\mathbf{h}(t)) \quad \forall i .
$$

By normalizing the unit of time so that $\lambda=1$, we obtain the following adjustment process:

$$
\dot{\mathbf{h}}(t)=\mathbf{F}(\mathbf{h}(t)) \equiv H \mathbf{P}(\mathbf{h}(t))-\mathbf{h}(t),
$$

where $\dot{\mathbf{h}}$ denotes the time derivative of $\mathbf{h}(t)$, and $\mathbf{P}(\mathbf{h}) \equiv\left[P_{0}(\mathbf{h}), P_{1}(\mathbf{h}), \ldots, P_{K-1}(\mathbf{h})\right]^{\mathrm{T}}$. For the specific functional form of $P_{i}(\mathbf{h}(t))$, we use the logit choice function:

$$
P_{i}(\mathbf{h}) \equiv \exp \left[\theta v_{i}(\mathbf{h})\right] / \sum_{j} \exp \left[\theta v_{j}(\mathbf{h})\right]
$$

where $\theta \in(0, \infty)$ is the parameter denoting the inverse of variance of the idiosyncratic tastes. This implies the assumption that the distributions of $\left\{\varepsilon_{i}^{(s)}, \forall s\right\}$ are Weibull distributions, which are identical and independent across regions (e.g., McFadden (1974), Anderson et al. (1992)). The adjustment process described by (3.14) and (3.15) is the logit dynamics, which has been studied in evolutionary game theory (e.g., Fudenberg and Levine (1998), Hofbauer and Sandholm (2002, 2007), and Sandholm (2009)).

Next, we define the long-run equilibrium and its stability. The long-run equilibrium is a stationary point of the adjustment process of (3.14):

Definition 3.1: The long-run equilibrium is defined as the distribution $\mathbf{h}^{*}$ that satisfies

$$
\mathbf{F}\left(\mathbf{h}^{*}\right) \equiv H \mathbf{P}\left(\mathbf{h}^{*}\right)-\mathbf{h}^{*}=\mathbf{0}
$$

Note that the heterogeneous consumer case includes the conventional homogeneous consumer case. Indeed, when $\theta \rightarrow \infty$, the condition given in (3.16) reduces to that for the homogeneous consumer case: 


$$
\left\{\begin{array}{lll}
V^{*}-v_{i}\left(\mathbf{h}^{*}\right)=0 & \text { if } & h_{i}^{*}>0 \\
V^{*}-v_{i}\left(\mathbf{h}^{*}\right) \geq 0 & \text { if } & h_{i}^{*}=0
\end{array},\right.
$$

where $V^{*}$ denotes the equilibrium utility.

We limit the area of concern to the neighborhood of $\mathbf{h}^{*}$, and define the stability of $\mathbf{h}^{*}$ in the sense of asymptotic stability, the precise definition of which is as follows.

Definition 3.2: A long-run equilibrium $\mathbf{h}^{*}$ is asymptotically stable if, for any $\varepsilon>0$, there is a neighborhood $N\left(\mathbf{h}^{*}\right)$ of $\mathbf{h}^{*}$ such that, for every $\mathbf{h}_{0} \in N\left(\mathbf{h}^{*}\right)$, the solution $\mathbf{h}(t)$ of (3.14) with an initial value $\mathbf{h}(0)=\mathbf{h}_{0}$ satisfies $\left\|\mathbf{h}(t)-\mathbf{h}^{*}\right\|<\varepsilon$ for any time $t \geq 0$, and $\lim _{t \rightarrow \infty} \mathbf{h}(t)=\mathbf{h}^{*}$. If equilibrium $\mathbf{h}^{*}$ is not asymptotically stable, it is unstable.

In dynamic system theory, $\mathbf{h}^{*}$ is asymptotically stable if all of the eigenvalues of the Jacobian matrix $\nabla \mathbf{F}(\mathbf{h}) \equiv\left[\partial F_{i}(\mathbf{h}) / \partial h_{j}\right]$ of the adjustment process of (3.14) have negative real parts; otherwise $\mathbf{h}^{*}$ is unstable (see, for example, Hirsch and Smale (1974)). This means that the asymptotic stability can be assessed by examining the following Jacobian matrix:

$$
\nabla \mathbf{F}(\mathbf{h})=H \mathbf{J}(\mathbf{h}) \nabla \mathbf{v}(\mathbf{h})-\mathbf{I},
$$

where $\mathbf{J}(\mathbf{h})$ and $\nabla \mathbf{v}(\mathbf{h})$ are $K$-by- $K$ matrices, the $(i, j)$ entries of which are $\partial P_{i}(\mathbf{v}) / \partial v_{j}$ and $\partial v_{i}(\mathbf{h}) / \partial h_{j}$, respectively. For the logit choice function of (3.15), it is easily verified that the former Jacobian matrix $\mathbf{J}(\mathbf{h})$ is expressed as

$$
\mathbf{J}(\mathbf{h})=\theta\left\{\operatorname{diag}[\mathbf{P}(\mathbf{h})]-\mathbf{P}(\mathbf{h}) \mathbf{P}(\mathbf{h})^{\mathrm{T}}\right\} .
$$

The latter Jacobian matrix $\nabla \mathbf{v}(\mathbf{h})$ is given by

$$
\nabla \mathbf{v}(\mathbf{h})=\nabla \mathbf{S}(\mathbf{h})+\sigma^{-1}\left\{\nabla \mathbf{w}^{(L)}(\mathbf{h})+\nabla \mathbf{w}^{(H)}(\mathbf{h})\right\},
$$

where $\quad \nabla \mathbf{S}=(\sigma-1)^{-1} \mathbf{M}^{\mathrm{T}}, \nabla \mathbf{w}^{(L)}=-\mathbf{M} \mathbf{M}^{\mathrm{T}} \quad, \quad \nabla \mathbf{w}^{(H)}=\mathbf{M}-\mathbf{M} \mathbf{H} \mathbf{M}^{\mathrm{T}} \quad, \quad$ and $\mathbf{H} \equiv \operatorname{diag}[\mathbf{h}]$.

\section{Spatial Discounting Matrix and Racetrack Economy}

In order to understand the bifurcation mechanism of the CP model, we need to know how the eigenvalues of the Jacobian matrix of the adjustment process of (3.14) depends on the bifurcation parameters (e.g., transportation $\operatorname{cost} \tau$ ). A combination of the racetrack economy (RE) defined in Section 4.1 and the resultant circulant properties of the spatial discounting matrix (SDM) shown in Section 4.2 greatly 
facilitates the analysis. Section 4.3 shows that the eigenvalues $\left\{g_{k}\right\}$ of the Jacobian matrix of the adjustment process can be expressed as simple functions of the eigenvalues $\left\{f_{k}\right\}$ of the SDM.

\subsection{Racetrack Economy}

Consider a racetrack economy (RE) in which $K$ regions $\{0,1,2, \ldots, K-1\}$ are equidistantly located on a circle of radius 1 . Let $t(i, j)$ denote the distance between regions $i$ and $j$. The distance between these two regions is defined as the minimum path length, i.e.,

$$
t(i, j)=(2 \pi / K) \cdot m(i, j)
$$

where $m(i, j) \equiv \min .\{|i-j|, K-|i-j|\}$. The set $\{t(i, j),(i, j=0,1,2, \ldots, K-1)\}$ of the distances can be represented by the spatial discounting matrix (SDM) D, entry $(i, j)$, $d_{i j}$, of which is given by

$$
d_{i j} \equiv \exp [-(\sigma-1) \cdot \tau \cdot t(i, j)]
$$

Defining the spatial discount factor (SDF) by

$$
r \equiv \exp [-(\sigma-1) \cdot \tau(2 \pi / K)]
$$

we can represent $d_{i j}$ as $r^{m(i, j)}$. It follows from the definition that the SDF $r$ is a monotonically decreasing function of the transportation cost parameter $\tau$, and hence the feasible range of the SDF (corresponding to $0 \leq \tau<+\infty)$ is given by $(0,1]$ : $\tau=0 \Leftrightarrow r=1$, and $\tau \rightarrow+\infty \Leftrightarrow r \rightarrow 0$.

As shown by Definitions (4.1) and (4.2), the SDM D in a racetrack economy is a circulant, which is constructed from the vector $\mathbf{d}_{0} \equiv\left[1, r, r^{2}, \ldots, r^{M}, \ldots, r^{2}, r\right]^{\mathrm{T}}$ (see, for example, Gray (2006) for the definition of circulants). This circulant property of the matrix $\mathbf{D}$ offers a key to understanding the essential properties of the CP model.

\subsection{Eigenvalue of the Spatial Discounting Matrix}

By exploiting the fact that the SDM D is a circulant matrix, we can easily obtain the eigenvalues $\mathbf{f} \equiv\left[f_{0}, f_{1}, \ldots, f_{K-1}\right]^{\mathrm{T}}$ of the SDM by discrete Fourier transformation (DFT). More specifically, the eigenvalues $\mathbf{f}$ are given by the following similarity transformation of $\mathbf{D}$ :

$$
\mathbf{Z}^{*} \mathbf{D Z}=\operatorname{diag}[\mathbf{f}]
$$

where $\mathbf{Z}$ is a DFT matrix, element $(j, k)$ of which is $z_{j k} \equiv w^{j k}(j, k=0,1, \ldots, K-1)$, $w \equiv e^{\mathrm{i}(2 \pi / K)}$, and $\mathbf{Z}^{*}$ denotes the conjugate transpose of $\mathbf{Z}$. Furthermore, (4.4) and the properties of a circulant (see, for example, Gray (2006)) show that DFT of the vector $\mathbf{d}_{0}$ (the first row vector of $\mathbf{D}$ ) directly yields the eigenvalues $\mathbf{f}$ : 


$$
\mathbf{f}=\mathbf{Z} \mathbf{d}_{0} .
$$

Since the entries of $\mathbf{d}_{0}$ satisfy $d_{0, m}=d_{0, K-m}(m=1,2, \ldots, M-1)$, where $M \equiv K / 2$, we see that the eigenvalues $\mathbf{f}$ are composed of $M-1$ roots with multiplicity 2 and two simple roots $(k=0, M)$. This together with the straightforward calculation of (4.5) yields the following lemma (proofs of lemmas are hereinafter given in the Appendices):

Lemma 4.1: The eigenvalues $\mathbf{f}$ of the SDM on the RE with $K$ regions are given by

$$
\begin{array}{ll}
f_{m}=c_{m}(r) R_{m}(r) & (m=0,1,2, \ldots, M), \\
f_{m}=f_{K-m} & (m=1,2, \ldots, M-1),
\end{array}
$$

where $\quad c_{m}(r) \equiv \frac{1-r^{2}}{1-2 r \cos (2 \pi m / K)+r^{2}}, \quad R_{m}(r) \equiv 1-(-1)^{m} r^{M} \quad(m=0,1,2, \ldots, M)$.

As shown in Section 4.3, the eigenvalues $\overline{\mathbf{f}}(r)$ of matrix $\mathbf{D} / d$, which can be obtained by normalizing the eigenvalues $\mathbf{f}(r)$ by $f_{0}=\mathbf{d}_{0} \cdot \mathbf{1} \equiv d$, play a key role in the analysis of the bifurcation from a uniform distribution $\overline{\mathbf{h}}$ (flat earth equilibrium). It follows from Lemma 4.1 that the eigenvalues $\overline{\mathbf{f}}(r)$ have the following properties.

Proposition 4.1: The normalized $S D M \mathrm{D} / d$ on the $R E$ with $K$ regions has the following eigenvector and the associated eigenvalues:

(i) the kth eigenvector $(k=0,1,2, \ldots, K-1)$ is given by the kth row vector, $\mathbf{z}_{k}$, of the discrete Fourier transformation (DFT) matrix $\mathbf{Z}$, that is,

$$
\mathbf{z}_{k} \equiv\left[1, w^{k}, w^{2 k}, \ldots, w^{k(K-1)}\right], \text { where } w \equiv e^{\mathrm{i}(2 \pi / K)} .
$$

(ii) the kth eigenvalue $\bar{f}_{k}(k=1,2, \ldots, K-1)$ is given by

$$
\begin{aligned}
& \bar{f}_{0}=1, \quad \bar{f}_{M}=\left(c_{M}(r)\right)^{2}, \\
& \bar{f}_{m}=\bar{f}_{K-m}=\left\{\begin{array}{ll}
c_{m}(r) c_{M}(r) & (m: \text { even }) \\
c_{m}(r) c_{M}(r) \varepsilon_{M}(r) & (m: \text { odd })
\end{array} \quad(m=1,2, \ldots, M-1),\right.
\end{aligned}
$$

where

$$
c_{M}(r)=(1-r) /(1+r), \quad \varepsilon_{M}(r) \equiv\left(1+r^{M}\right) /\left(1-r^{M}\right) .
$$

(iii) $\bar{f}_{m}(m=1,2, \ldots, M)$ is a monotonically decreasing function of $r$ for $\forall r \in[0,1)$.

(iv) $\left\{\begin{array}{l}\max _{m} \cdot\left\{\bar{f}_{m}(r)\right\}=\bar{f}_{0}(r)=1 \\ \min _{m} \cdot\left\{\bar{f}_{m}(r)\right\}=\bar{f}_{M}(r)=\left(c_{M}(r)\right)^{2}\end{array}\right.$ for $\forall r \in[0,1)$. 
(v) $\left\{\begin{array}{l}1=\bar{f}_{0}>\bar{f}_{2}>\cdots>\bar{f}_{2 k}>\cdots>\bar{f}_{M}=c_{M}^{2} \\ 1 \geq \bar{f}_{1}>\bar{f}_{3}>\cdots>\bar{f}_{2 k+1}>\cdots>\bar{f}_{M-1}>c_{M}^{2} \varepsilon_{M}\end{array}\right.$ for $\forall r \in[0,1)$

Figure 1 illustrates the properties of the eigenvalues $\overline{\mathbf{f}}$ in Proposition 4.1 for the case of $K=16(M=K / 2=8)$. For convenience in the bifurcation analysis in later sections, we depict the eigenvalues as functions of the transportation cost parameter $\tau$. It follows from Definition (4.3) that the SDF $r \in[0,1)$ is a monotonically decreasing function of $\tau$ (see Definition (4.3)), and that $\tau=0 \Leftrightarrow r=1$ and $\tau \rightarrow+\infty \Leftrightarrow r \rightarrow 0$. This, together with (iii) in the proposition, indicate that $\bar{f}_{m}$ is a monotonically increasing function of $\tau$ and that $\tau=0 \Leftrightarrow \bar{f}_{m}=0$ and $\tau \rightarrow+\infty \Leftrightarrow \bar{f}_{m} \rightarrow 1$.

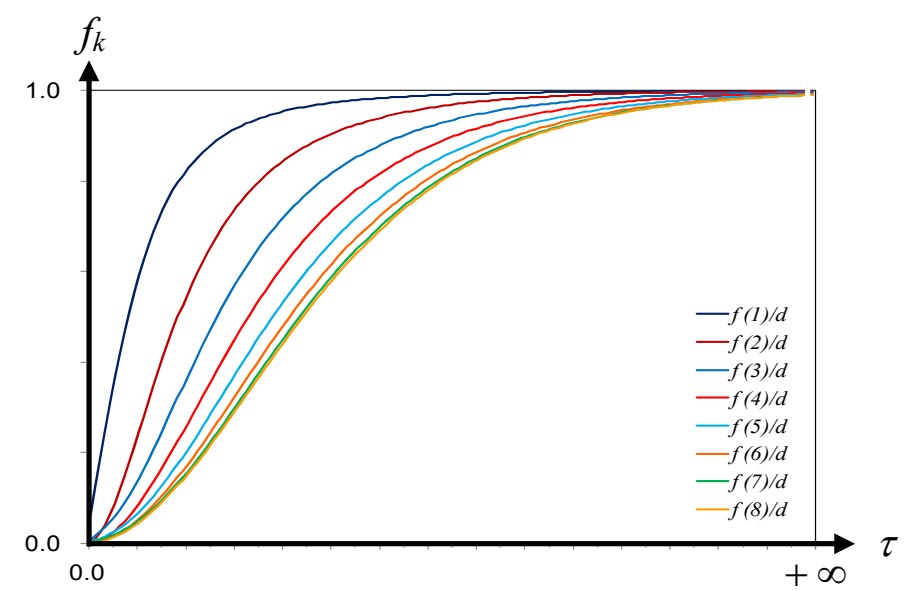

Figure 1. Eigenvalues $\bar{f}_{m}(m=0,1,2, . ., 8)$ of $\mathbf{D} / d$ for $K=16$ as functions of transportation cost parameter $\tau$

\subsection{Eigenvalues of the Jacobi Matrices}

The eigenvalues $\mathbf{g}$ of the Jacobian matrix $\nabla \mathbf{F}(\mathbf{h})$ at an arbitrary distribution $\mathbf{h}$ of the skilled labor cannot be obtained without resorting to numerical techniques. It is, however, possible at some symmetric distributions $\overline{\mathbf{h}}$ to obtain analytical expressions for the eigenvalues $\mathbf{g}$ of the Jacobian. In particular, a uniform distribution of skilled workers (flat earth equilibrium), $\overline{\mathbf{h}} \equiv[h, h, \ldots, h, h]$ and $h \equiv H / K$, which has intrinsic significance in examining the emergence of agglomeration, gives us the simplest example for illustrating this fact. At the uniform configuration $\overline{\mathbf{h}}$, the Jacobian matrix of the adjustment process:

$$
\nabla \mathbf{F}(\overline{\mathbf{h}})=H \mathbf{J}(\overline{\mathbf{h}}) \nabla \mathbf{v}(\overline{\mathbf{h}})-\mathbf{I}
$$

reduces to a circulant, as shown in the following lemmas.

Lemma 4.2: The Jacobian matrices $\mathbf{J}(\overline{\mathbf{h}})$ and $\nabla \mathbf{v}(\overline{\mathbf{h}})$ at $\overline{\mathbf{h}}$ are given by 


$$
\mathbf{J}(\overline{\mathbf{h}})=(\theta / K)\left(\mathbf{I}-\mathbf{1 1}^{\mathrm{T}} / K\right),
$$

$$
\nabla \mathbf{v}(\overline{\mathbf{h}})=h^{-1}\left\{b[\mathbf{D} / d]-a[\mathbf{D} / d]^{2}\right\},
$$

where $a \equiv \sigma^{-1}\left(1+h^{-1}\right), \quad b \equiv(\sigma-1)^{-1}+\sigma^{-1}, \mathbf{1} \equiv[1,1, \ldots, 1]^{\mathrm{T}}$.

Lemma 4.3: The Jacobian matrices $\mathbf{J}(\overline{\mathbf{h}}), \nabla \mathbf{v}(\overline{\mathbf{h}})$, and $\nabla \mathbf{F}(\overline{\mathbf{h}})$ at $\overline{\mathbf{h}}$ are circulants.

The fact that $\nabla \mathbf{F}(\overline{\mathbf{h}})$ is a circulant allows us to obtain the eigenvalues $\mathbf{g}$ of $\nabla \mathbf{F}(\overline{\mathbf{h}})$ using the DFT matrix Z. Specifically, both sides of (4.9) can be diagonalized by the similarity transformation based on $\mathbf{Z}$ :

$$
\operatorname{diag}[\mathbf{g}]=H \operatorname{diag}[\boldsymbol{\delta}] \operatorname{diag}[\mathbf{e}]-\operatorname{diag}[\mathbf{1}],
$$

where $\delta$ and $\mathbf{e}$ are the eigenvalues of $\mathbf{J}(\overline{\mathbf{h}})$ and $\nabla \mathbf{v}(\overline{\mathbf{h}})$, respectively. The two eigenvalues, $\delta$ and $\mathbf{e}$, in the right-hand side of (4.12) are easily obtained as follows. The former eigenvalues $\delta$ are obtained by DFT of the first row vector of $\mathbf{J}(\overline{\mathbf{h}})$ in (4.11):

$$
\delta=(\theta / K)[0,1,1,1, \ldots, 1]^{\mathrm{T}} .
$$

As for the latter eigenvalues e, note that $\nabla \mathbf{v}(\overline{\mathbf{h}})$ in (4.10) consists of additions and multiplications of the circulant $\mathbf{D} / d$. This implies that the eigenvalues $\mathbf{e}$ are represented as functions of the eigenvalues $\overline{\mathbf{f}}$ of $\mathbf{D} / d$ :

$$
\mathbf{e}=h^{-1}\left\{b[\overline{\mathbf{f}}]-a[\overline{\mathbf{f}}]^{2}\right\},
$$

where $[\mathbf{x}]^{2} \equiv[\mathbf{x}] \cdot[\mathbf{x}]$, and $[\mathbf{x}] \cdot[\mathbf{y}]$ denotes the component-wise products of vectors $\mathbf{x}$ and $\mathbf{y}$. Thus, we arrive at the following proposition for the eigenvalues of $\nabla \mathbf{F}(\overline{\mathbf{h}})$ :

Proposition 4.2: Consider a uniform distribution $\overline{\mathbf{h}}$ of skilled workers in the RE with $K$ regions. The Jacobian matrix $\nabla \mathbf{F}(\overline{\mathbf{h}})$ of the adjustment process of (3.14) at $\overline{\mathbf{h}}$ has the following eigenvector and associated eigenvalues:

(i) the $k$ th eigenvector $(k=1,2, \ldots, K-1)$ is given by the $k$ th row vector, $\mathbf{z}_{k}$, of the discrete Fourier transformation (DFT) matrix $\mathbf{Z}$.

(ii) the $k$ th eigenvalue $g_{k}(k=1,2, \ldots, K-1)$ is given by a quadratic function of the $k$ th eigenvalue $\bar{f}_{k}$ of the spatial discounting matrix $\mathbf{D} / d$ :

$$
\begin{aligned}
& g_{k}=\theta G\left(\bar{f}_{k}\right) \quad(k=1,2, \ldots, K-1), \quad g_{0}=-1, \\
& G(x) \equiv b x-a x^{2}-\theta^{-1},
\end{aligned}
$$

where $a \equiv \sigma^{-1}\left(1+h^{-1}\right), b \equiv(\sigma-1)^{-1}+\sigma^{-1}$. 
The eigenvectors $\left\{\mathbf{z}_{k}(k=1,2, \ldots, K-1)\right\}$ in the first part of Proposition 4.2 represent the agglomeration patterns of skilled workers by the configuration pattern of the entries. For example, all entries of $\mathbf{z}_{0}$ are equal to 1 , and the entry pattern of $\mathbf{z}_{0}=[1,1, \ldots, 1]$ corresponds to the state in which skilled workers are uniformly distributed among $K$ regions, whereas $\mathbf{z}_{M}=[1,-1,1,-1 \ldots,-1]$, where the alternating sequence of 1 and -1 , represents an $M$-centric agglomerate pattern in which skilled workers reside in $M=K / 2$ regions alternately. Similarly, $\mathbf{z}_{M / 2}$ correspond to an $M / 2$-centric pattern.

The eigenvalues $g_{k}$ in the second part of the proposition can be interpreted as the strength of net agglomeration force that leads the uniform distribution to the direction of the $k$ th agglomeration pattern (i.e., $\mathbf{z}_{k}$ ). The term net agglomeration force refers to the net effect of the agglomeration force minus the dispersion force. Specifically, these forces correspond to $b x$ and $a x^{2}$, respectively, in (4.13b). As is clear from the derivation of the eigenvalues $\mathbf{g}$, the term $b x$ stems from $\nabla S$ and the first term of $\nabla \mathbf{w}^{(H)}$, which represent forward linkage (or price-index effect) and backward linkage (or market access effect of firms), respectively. Thus, the term $b x$ represents the agglomeration force induced by the increase in the variety of products that would be realized when the uniform distribution $\overline{\mathbf{h}}$ deviates to agglomeration pattern $\mathbf{z}_{k}$. The term $a x^{2}$, which stems from $\nabla \mathbf{w}^{(L)}$ and the second term of $\nabla \mathbf{w}^{(H)}$, represents the dispersion force due to increased market competition (market crowding effect) in the agglomerated pattern $\mathbf{z}_{k}$.

\section{Uniform Distribution and Agglomeration}

\subsection{The First Bifurcation - Emergence of Agglomeration}

We are now ready to examine the emergence of agglomeration in the CP model. In this and subsequent sections, we consider the bifurcation process of the equilibrium, where the value of the transportation cost $\tau$ steadily decreases from a very high value.

In order for the bifurcation from the flat earth equilibrium to occur with the changes in $\tau$, either of the eigenvalues $\left\{g_{k}, \forall k \neq 0\right\}$ must changes sign. Since the eigenvalues $g_{k}$ are given by $G\left(f_{k}\right)$, changes in sign indicate that there should exist real solutions for the following quadratic equation with respect to $f_{k}$ :

$$
G\left(f_{k}\right)=0
$$

Moreover, the solutions must lie in the interval $[0,1)$, which is the possible range of the eigenvalue $f_{k}$. These conditions lead to the following proposition: 
Proposition 5.1: Starting from a very high value of the transport cost $\tau$, we steadily decrease the value of $\tau$. In order for a bifurcation from a uniform equilibrium to some agglomeration to occur in the RE, the parameters of the CP model should satisfy

$$
\Theta \equiv b^{2}-4 a \theta^{-1} \geq 0 \text { and } b+\sqrt{\Theta} \leq 2 a .
$$

The first inequality in Proposition 5.1 is the condition for the existence of real solutions of (5.1) with respect to $f_{k}$. This condition is not necessarily satisfied for cases in which the heterogeneity of consumers is very large (i.e., $\theta$ is very small), which implies that no agglomeration occurs in such cases. The second inequality, which stems from the requirement that solutions of (5.1) be less than 1, corresponds to the no-black-hole condition, as described in the literature dealing with the two-region CP model. For cases in which the no-black-hole condition is not satisfied, the eigenvalues $\left\{g_{k}, \forall k \neq 0\right\}$ are positive even when the transportation cost $\tau$ is very high, which means that the uniform distribution $\overline{\mathbf{h}}$ cannot be a stable equilibrium.

In the following analyses, we assume that the parameters $(h, \sigma, \theta)$ of the $\mathrm{CP}$ model satisfy (5.2) in Proposition 5.1. We then have two real solutions for (5.1) with respect to $f_{k}$ :

$$
x_{+}^{*} \equiv(b+\sqrt{\Theta}) /(2 a) \text { and } x_{-}^{*} \equiv(b-\sqrt{\Theta}) /(2 a)
$$

Each of the solutions $x_{ \pm}^{*}$ represents a critical value (break point) at which a bifurcation from the flat earth equilibrium $\overline{\mathbf{h}}$ occurs if we regard the eigenvalue $f_{k}$ as a bifurcation parameter.

Since we are interested in the process of decreasing the value of $\tau$, it is more meaningful to express the break point in terms of $\tau$ (rather than in terms of $f_{k}$ ). For this purpose, we define the inverse function, $\tau_{k}(\cdot)$, of the eigenvalue $\bar{f}_{k}(\tau)$ as a function of $\tau$ :

$$
\tau^{*}=\tau_{k}\left(x^{*}\right) \Leftrightarrow x^{*}=\bar{f}_{k}\left(\tau^{*}\right) .
$$

The inverse $\tau_{k}(\cdot)$ is a monotonically increasing function because $\bar{f}_{k}(\cdot)$ is a monotonically increasing function of $\tau$ (see Figure 1).

Now suppose that the transportation cost is initially large enough for the condition $\tau>\tau_{k}\left(x_{+}^{*}\right) \forall k \neq 0$ to hold. From Definition (5.4) of the inverse function $\tau_{k}(\cdot)$, this is equivalent to

$$
\bar{f}_{k}(\tau)>x_{+}^{*} \quad \forall k \neq 0 .
$$

Since the eigenvalue $g_{k}$ is a concave function $G$ of $\bar{f}_{k}$ (see Proposition 4.2), the condition given in (5.5) implies that $g_{k}<0 \forall k \neq 0$ holds, and hence the flat earth 
equilibrium is stable. We then consider what happens when the value of $\tau$ steadily decreases. The decrease in $\tau$ leads to a state in which $\tau \leq \tau_{k}\left(x_{+}^{*}\right)$ holds for some $k$. This can be equivalently represented as

$$
\bar{f}_{k}(\tau) \leq x_{+}^{*} \quad \exists k
$$

which means that $g_{k} \geq 0 \quad \exists k$, and, therefore, the flat earth equilibrium is unstable. While (5.6) does not explicitly show which of the eigenvalues $\left\{\bar{f}_{k}(\tau)\right\}$ first satisfies this condition, Proposition 4.1 shows that the $M$ th eigenvalue first reaches the critical value $x_{+}^{*}$ because

$$
\bar{f}_{M}(\tau)=\min _{k} .\left\{\bar{f}_{k}(\tau)\right\} \quad \text { for } \forall \tau \in[0,+\infty) .
$$

Accordingly, the critical value of $\tau$ at which the first bifurcation occurs is given by

$$
\tau_{+}^{*} \equiv \max _{k}\left\{\tau_{k}\left(x_{+}^{*}\right)\right\}=\tau_{M}\left(x_{+}^{*}\right) .
$$

The above procedure for obtaining the break point (at which the bifurcation occurs) can also be illustrated graphically, as shown in Figure 2. In the course of steadily decreasing the value of $\tau$, the bifurcation from the flat earth equilibrium occurs when either of $\bar{f}_{k}(\tau)$ first takes a value less than $x_{+}^{*}$. Figure 2 shows that this occurs at the point of intersection $\mathrm{C}_{+}$of the horizontal line $\ell_{+}$(which represents $\bar{f}_{k}=x_{+}^{*}$ ) and the curve $\bar{f}_{M}(\tau)$ (which is the minimum of $\left\{\bar{f}_{k}(\tau)\right\}$ ). This figure also shows that $\mathrm{C}_{+}$is characterized as the point at which the maximum of $\left\{\tau_{k}\left(x_{+}^{*}\right)\right\}$ is attained. This yields (5.7) for the critical value of $\tau$.

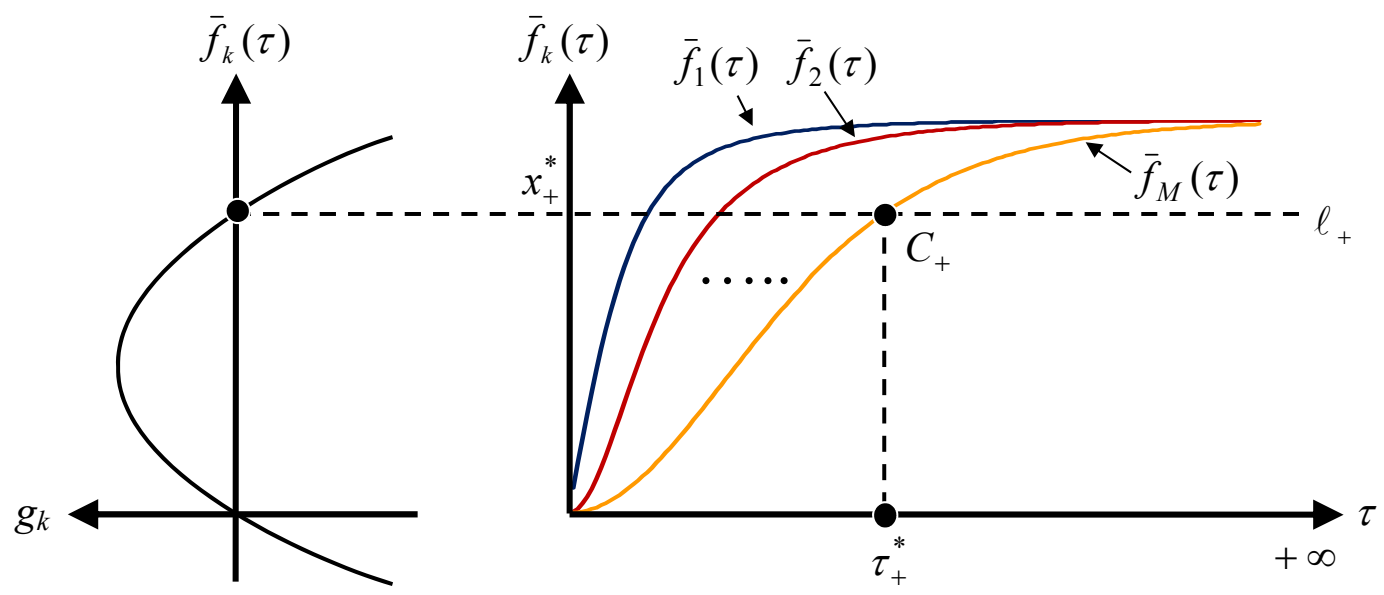

Figure 2. First break point in the course of decreasing $\tau$

The fact that the $M$ th eigenvalue first reaches the critical value $x_{+}^{*}$ also enables us to identify the associated agglomeration pattern that emerges at the first bifurcation. 
Recall here that the equilibrium moves in the direction of the eigenvector for which the associated eigenvalue reaches the critical value. As stated in Proposition 4.2, this direction is the $M$ th eigenvector $\mathbf{z}_{M}=[1,-1, \ldots, 1,-1]$. Therefore, the pattern of agglomeration that first emerges is

$$
\mathbf{h}=\overline{\mathbf{h}}+\delta \mathbf{z}_{M}=[h+\delta, h-\delta, \ldots, h+\delta, h-\delta] \quad(0 \leq \delta \leq h),
$$

in which skilled workers agglomerate in alternate regions.

Finally, note that (5.7) implicitly indicates how the changes in the values of the CP model parameters affect the critical value $\tau_{+}^{*}$. More specifically, (5.7) is equivalent to $x_{+}^{*}=f_{M}\left(r\left(\tau_{+}^{*}\right)\right)$, which yields the critical value in terms of the SDF:

$$
r\left(\tau_{+}^{*}\right)=\left(1-\left(x_{+}^{*}\right)^{1 / 2}\right) /\left(1+\left(x_{+}^{*}\right)^{1 / 2}\right) .
$$

This yields the relationship between $\tau_{+}^{*}$ and the $\mathrm{CP}$ model parameters $(h, \sigma, \theta)$ because $x_{+}^{*}$ is explicitly represented as a function of $(h, \sigma, \theta)$ in (5.3) and the SDF $r$ is a simple exponential function of $\tau$, as defined in (4.3). Thus, we can characterize the bifurcation from the uniform distribution as follows.

Proposition 5.2: Suppose that the CP model satisfies the conditions described in Proposition 5.1. Starting from a state in which the value of the transportation cost $\tau$ is large enough for the uniform distribution $\overline{\mathbf{h}}$ to be a stable equilibrium, we consider the process in which the value of $\tau$ steadily decreases.

(i) The net agglomeration force (i.e., the eigenvalue) $g_{k}$ for each agglomeration pattern (i.e., the eigenvector) $\mathbf{z}_{k}$ increases with the decrease in $\tau$, and the uniform distribution becomes unstable at the break point $\tau=\tau_{+}^{*}$ given by (5.3) and (5.7).

(ii) The critical value $\tau_{+}^{*}$ decreases as a) the heterogeneity of skilled workers (in location choice) becomes smaller (i.e., $\theta$ is large), b) the number of skilled workers relative to that of the unskilled workers becomes larger (i.e., $h$ is large), and c) the elasticity of substitution between two varieties becomes smaller (i.e., $\sigma$ is small).

(iii) The pattern of agglomeration that first emerges is $\mathbf{h}=[h+\delta, h-\delta, \cdots, h+\delta, h-\delta](0 \leq \delta \leq h)$, in which skilled workers agglomerate in alternate regions.

\subsection{The Last Bifurcation - Collapse of Agglomeration}

In Section 5.1, we have derived two critical values (with respect to the eigenvalue $\left.f_{k}\right), x_{+}^{*}$ and $x_{-}^{*}$, at each of which a bifurcation from the uniform distribution $\overline{\mathbf{h}}$ occurs, and only the properties of the bifurcation at the former critical value $\left(x_{+}^{*}\right)$ have 
been shown. We shall examine the bifurcation at the latter critical value $\left(x_{-}^{*}\right)$. We discuss two cases, A and B. In Case A, consumers are homogeneous with respect to location choice (i.e., $\theta \rightarrow+\infty$ ), and in Case $\mathrm{B}$, consumers are inhomogeneous (i.e., $\theta$ is finite). As shown below, these two cases exhibit significantly different properties of the bifurcation at the critical value $x_{-}^{*}$.

A) For the homogeneous consumer case, $\Theta=b^{2}$ and the critical value given by (5.3) reduces to the following simpler expression:

$$
\lim _{\theta \rightarrow+\infty} x_{ \pm}^{*}(\theta)=b / a \text { or } 0 \text {. }
$$

Substituting this into the definition of $\tau_{k}(\cdot)$, we see that $\tau_{k}\left(x_{-}^{*}\right)=\tau_{k}(0)=0 \forall k \neq 0$. Therefore, the critical value $\tau_{-}^{*}$ for the bifurcation from some agglomeration to the flat earth equilibrium is zero:

$$
\lim _{\theta \rightarrow+\infty} \tau_{-}^{*}=\min _{k} .\left\{\tau_{k}(0)\right\}=0 .
$$

This means that at least one of the eigenvalues $\left\{g_{k}\right\}$ is always positive for the interval $\left(0, \tau_{M}\left(x_{+}^{*}\right)\right]$ of $\tau$ (see Figure 3). That is, no matter how small the transportation cost, agglomeration never collapses, except for the minimum limit of $\tau=0$.

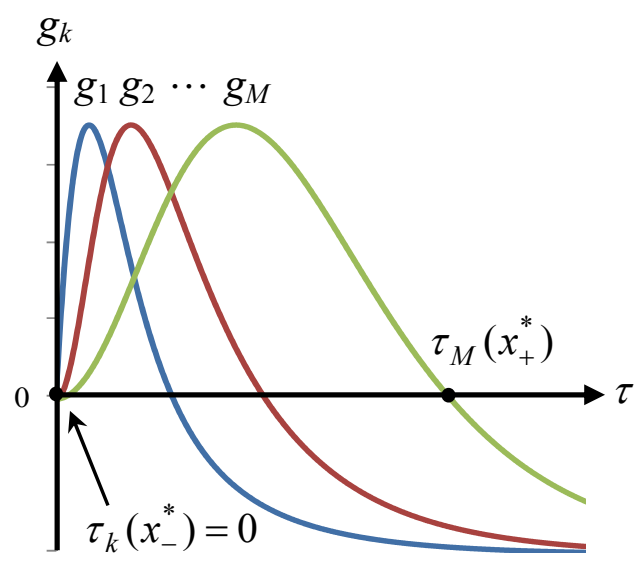

A) Homogeneous consumer case

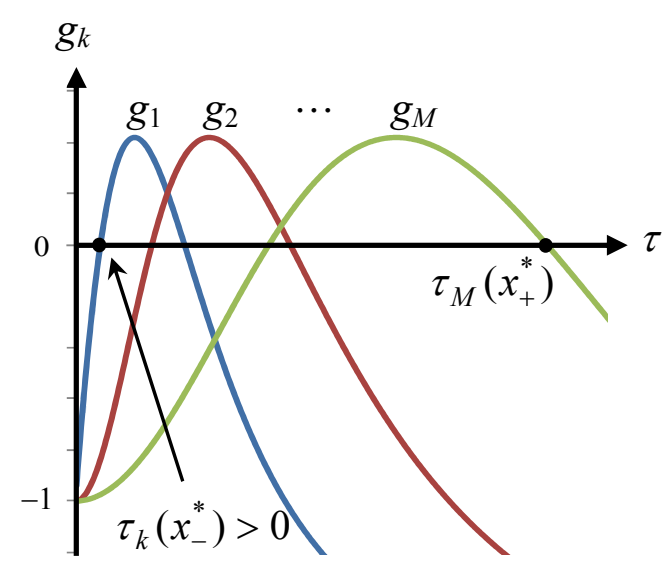

B) Heterogeneous consumer case

Figure 3. Stability and eigenvalue $g_{k}$

B) For the case in which consumers are heterogeneous, we see from (5.3) that the critical value $x_{-}^{*}$ is always positive regardless of the values of the CP model parameters, and thus $\tau_{k}\left(x_{-}^{*}\right)>0 \forall k$. This means that the net agglomeration force $g_{k}$ can be negative on the interval $\left(0, \tau_{k}\left(x_{-}^{*}\right)\right]$ of $\tau$ (see Figure 3). Accordingly, in the course of decreasing $\tau$, a bifurcation from some agglomeration to the uniform distribution (re-dispersion) $\overline{\mathbf{h}}$ necessarily occurs at the critical value:

$$
\tau_{-}^{*}=\min _{k} .\left\{\tau_{k}\left(x_{-}^{*}\right)\right\}>0 .
$$


That is, the CP economy with heterogeneous consumers necessarily moves from agglomeration to dispersion when the transportation cost $\tau$ decreases to the critical value. This is a generalized result of the conventional bell-shaped bifurcation in the two-region CP model with idiosyncratic differences in location choice (e.g., Tabuchi (1998), Helpman (1998), Murata (2003)).

It is worthwhile to examine the properties of the re-dispersion more closely. First, it can be deduced that, even if the consumers are homogeneous, the re-dispersion occurs when some dispersion forces (e.g., land rent) that increase with agglomeration are introduced into the CP model. This is because the necessary and sufficient condition for the occurrence of the re-dispersion is that the parameters of the $\mathrm{CP}$ model satisfy $x_{-}^{*}>0$. This requirement can be satisfied by either adding an extra constant term in the function $G$ in (4.13) or introducing heterogeneity in location choice (i.e., $\theta$ is finite).

Second, the re-dispersion can be observed not only at the critical value $\tau_{-}^{*}$ but also in the course of evolving agglomeration. The re-dispersion at $\tau_{-}^{*}$ is the last among multiple re-dispersions in the course of decreasing $\tau$. The mechanism of how such repetitions of agglomeration and dispersion may occur can best be explained by the example in Figure $4^{11}$. Each curve in this figure represents the eigenvalue $f_{k}$ at the uniform distribution $\overline{\mathbf{h}}$ as a function of $\tau$. Recall that a bifurcation from agglomeration to dispersion occurs when all of the eigenvalues $\left\{g_{k}\right\}$ at $\overline{\mathbf{h}}$ become negative. We can see from the figure that this actually occurs twice during the process of decreasing $\tau$. Specifically, the first re-dispersion is observed at the boundary $\tau_{-}^{* *}$ between ranges $\mathrm{A}$ and $\mathrm{B}$. Some agglomeration is present in range $\mathrm{A}$, because either of $\left\{\bar{f}_{k}, \forall k \neq 0,1\right\}$ is in $\left[x_{-}^{*}, x_{+}^{*}\right]$, and hence either of the eigenvalues $\left\{g_{k}, \forall k \neq 0,1\right\}$ at $\overline{\mathbf{h}}$ is positive, but $\overline{\mathbf{h}}$ become stable below the boundary $\tau_{-}^{* *}$ (range B) because all of the eigenvalues $\left\{g_{k}\right\}$ at $\overline{\mathbf{h}}$ are negative. (Note that $\bar{f}_{1}>x_{+}^{*}$ and $\bar{f}_{k}<x_{-}^{*} \forall k \neq 0,1$, which means that none of $\left\{\bar{f}_{k}\right\}$ is in $\left[x_{-}^{*}, x_{+}^{*}\right]$.) Note here that some agglomeration again emerges in range $\mathrm{C}$ because $\bar{f}_{1}=\bar{f}_{15}$ are in $\left[x_{-}^{*}, x_{+}^{*}\right]$, and hence the eigenvalues $g_{1}=g_{15}$ at $\overline{\mathbf{h}}$ are positive. This leads to the second re-dispersion at the boundary $\tau_{-}^{*}$ between ranges $\mathrm{C}$ and $\mathrm{D}$, and $\overline{\mathbf{h}}$ again becomes stable below the boundary $\tau_{-}^{*}$ (range D) because all of the eigenvalues $\left\{g_{k}\right\}$ at $\overline{\mathbf{h}}$ are negative. (Note that $\bar{f}_{k}<x_{-}^{*} \forall k \neq 0$, which means that none of $\left\{\bar{f}_{k}\right\}$ is in $\left[x_{-}^{*}, x_{+}^{*}\right]$.)

The discussion thus far can be summarized as the following proposition:

\footnotetext{
${ }^{11}$ Detailed discussions on the economic implications/interpretations of this phenomenon are relegated to Akamatsu, Takayama, and Sugasawa (2009) because the purpose of the present paper is to demonstrate a method by which to analyze the CP models.
} 
Proposition 5.3: Starting with an agglomeration state, we consider the process in which the value of the transportation cost $\tau$ decreases.

(i) The net agglomeration force $g_{k}$ for each $\mathbf{z}_{k}$ monotonically decreases with the decrease in $\tau$ after a monotonic increase (i.e., $g_{k}$ is a unimodal function of $\tau$ ).

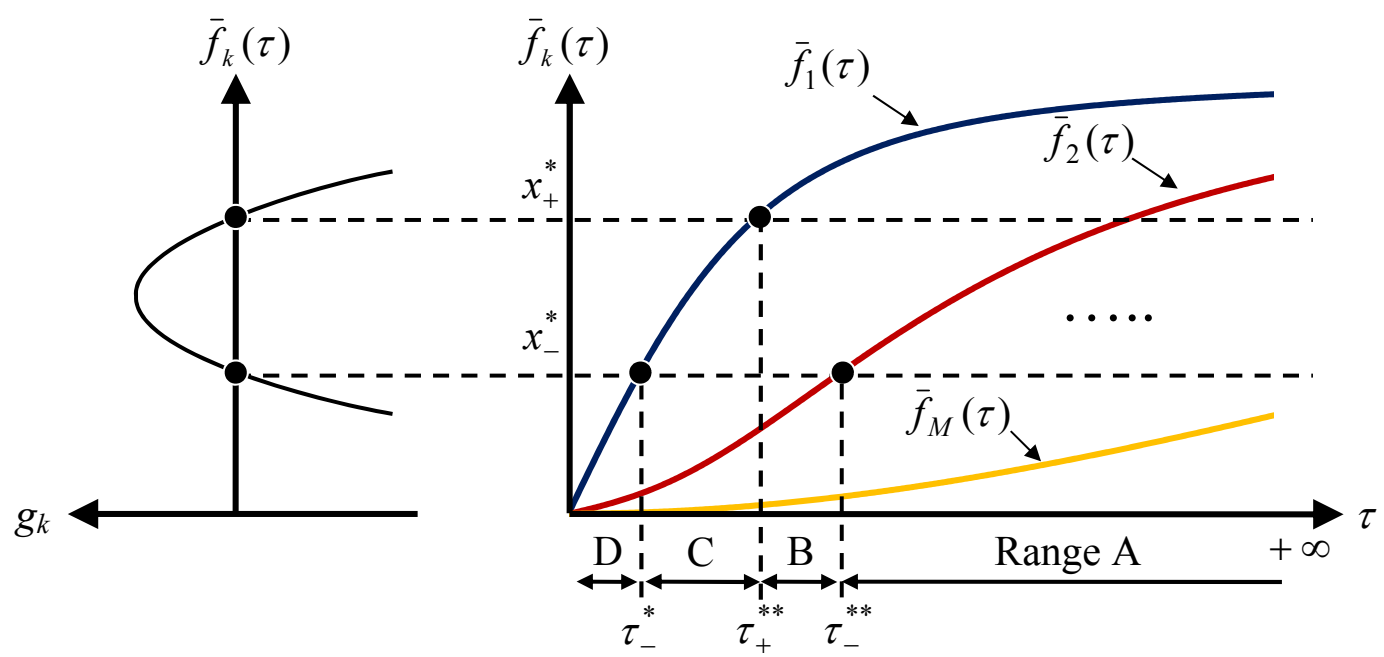

Figure 4. Repetitions of agglomeration and dispersion

(ii) For the CP model with homogeneous consumers, all of the net agglomeration forces reach zero only at the limit of $\tau=0$. That is, the agglomeration equilibrium never reverts to the uniform distribution equilibrium during the course of decreasing $\tau$.

(iii) For the CP model with heterogeneous consumers, all of the net agglomeration forces reach zero at a strictly positive value of $\tau$. That is, a bifurcation from some agglomeration to the uniform distribution equilibrium (re-dispersion) occurs at $\tau=\tau_{-}^{*}$, where $\tau_{-}^{*}$ is given by (5.10) and (5.3). Furthermore, repetitions of agglomeration and dispersion may be observed (see Figure 4).

\section{Evolution of Agglomeration Patterns}

In conventional two-region CP models, steady decreases in transportation cost lead to the occurrence of a bifurcation from the uniform distribution $\overline{\mathbf{h}}$ to a monocentric agglomeration. In the multi-regional $\mathrm{CP}$ model, however, the first bifurcation shown in Section 5 does not directly branch to the monocentric agglomeration. Instead, further bifurcations leading to a more concentrated pattern can repeatedly occur. In this section, we examine such evolution of agglomeration after the first bifurcation, restricting ourselves to the homogeneous consumer case (i.e., $\theta \rightarrow+\infty$ ). First, in 
Section 6.1 , it is shown that a second bifurcation from $\overline{\mathbf{h}}$ indeed occurs, which suggests a general rule of the agglomeration process whereby the multi-regional CP economy exhibits the spatial period doubling bifurcation (SPDB). In Section 6.2, a proof of this conjecture, including a general analytical formula to predict the occurrence of the SPDB, is presented. In order to illustrate the theoretical results, a number of numerical examples are presented in Section 6.3.

\subsection{The Second Bifurcation}

As we decrease the value of $\tau$ from the break point $\tau=\tau_{+}^{*}$ (at which the first bifurcation occurs), the deviation $\delta$ from the uniform distribution $\overline{\mathbf{h}}$ increases monotonically. This eventually leads to an $M$-centric pattern, $\mathbf{h}^{*}=[2 h, 0,2 h, 0, \ldots, 2 h, 0]$, where skilled workers equally exist only in the alternate two regions. In other words, skilled workers/firms in half of the regions are completely absorbed into the neighboring core regions (in which skilled workers agglomerate) in $\mathbf{h}^{*}$. The fact that the $M$-centric pattern $\mathbf{h}^{*}$ may exist as an equilibrium of the $\mathrm{CP}$ model can be confirmed by examining the sustain point for $\mathbf{h}^{*}$. The sustain point is the value of $\tau$ below which the equilibrium condition for $\mathbf{h}^{*}$,

$$
v_{0}\left(\mathbf{h}^{*}\right)=v_{2}\left(\mathbf{h}^{*}\right)=\max _{k}\left\{v_{k}\left(\mathbf{h}^{*}\right)\right\}
$$

is satisfied. As shown in Appendix 3, the condition of (6.1) indeed holds for any $\tau$ smaller than $\tau_{01}^{*}$, which is the sustain point for $\mathbf{h}^{*}$.

After the emergence of the $M$-centric pattern $\mathbf{h}^{*}=[2 h, 0,2 h, 0, \ldots, 2 h, 0]$, further decreases in $\tau$ below the sustain point can lead to further bifurcations (i.e., $\mathbf{h}^{*}$ become unstable). In order to investigate such a possibility, we need to obtain the eigenvectors and the associated eigenvalues for the Jacobian matrix of the adjustment process at $\mathbf{h}^{*}$ :

$$
\nabla \mathbf{F}\left(\mathbf{h}^{*}\right)=H \mathbf{J}\left(\mathbf{h}^{*}\right) \nabla \mathbf{v}\left(\mathbf{h}^{*}\right)-\mathbf{I}
$$

A seeming difficulty encountered in obtaining the eigenvalues is that, unlike the Jacobian $\nabla \mathbf{F}(\overline{\mathbf{h}})$ at $\overline{\mathbf{h}}$, the Jacobian $\nabla \mathbf{F}\left(\mathbf{h}^{*}\right)$ at $\mathbf{h}^{*}$ is no longer a circulant matrix. This is due to the loss of symmetry in the configuration $\mathbf{h}^{*}$ of skilled workers, which leads to $\mathbf{J}\left(\mathbf{h}^{*}\right)$ and $\nabla \mathbf{v}\left(\mathbf{h}^{*}\right)$ not being circulant matrices. However, it is still possible to find a closed-form expression for the eigenvalues of $\nabla \mathbf{F}\left(\mathbf{h}^{*}\right)$ based on the fact that $\mathbf{h}^{*}$ has partial symmetry and the submatrices of $\mathbf{J}\left(\mathbf{h}^{*}\right)$ and $\nabla \mathbf{v}\left(\mathbf{h}^{*}\right)$ are circulants.

In order to exploit the symmetry remaining in the $M$-centric pattern $\mathbf{h}^{*}$, we begin by dividing the set $C=\{0,1,2, \ldots, K-1\}$ of regions into two subsets, namely, the subset $C_{0}=\{0,2, \ldots, K-2\}$ of regions with skilled workers and the subset $C_{1}=\{0,3, \ldots, K-1\}$ of regions without skilled workers. Corresponding to this division of the set of regions, 
we consider the following permutation $\sigma$ of the set $C$ :

$$
\sigma:\left[\begin{array}{ccccc:cccc}
0 & 1 & 2 & \cdots & M-1 & M & M+1 & \cdots & K-1 \\
0 & 2 & 4 & \cdots & K-2 & 1 & 3 & \cdots & K-1
\end{array}\right],
$$

so that the first half elements $\{\sigma(0), \sigma(1), \ldots, \sigma(M-1)\}$ and the second half elements $\{\sigma(M), \sigma(M+1), \ldots, \sigma(K-1)\}$ of the set $C^{P}=\{\sigma(0), \sigma(1), \ldots, \sigma(K-1)\}$ correspond to the sub-sets $C_{0}$ and $C_{1}$, respectively. For this permutation, we define the associated $K$-by- $K$ permutation matrix $\mathbf{P}_{(1)}$ :

$$
\mathbf{P}_{(1)} \equiv\left[\begin{array}{c}
\mathbf{P}_{(1)}^{e} \\
\mathbf{P}_{(1)}^{o}
\end{array}\right],
$$

where $\mathbf{P}_{(1)}^{e}$ and $\mathbf{P}_{(1)}^{o}$ are $M$-by- $K$ matrices. The $(i, j)$ element of $\mathbf{P}_{(1)}^{e}$ is 1 if $j=2 i$ $(i=0,1,2, \ldots, M-1)$, and is otherwise 0 , whereas that of $\mathbf{P}_{(1)}^{o}$ is 1 if $j=2 i+1$, and is otherwise 0 . It is readily verified that, for a $K$-by- $K$ matrix $\mathbf{A}$, the $(i, j)$ element of which is $a_{i j}, \mathbf{P}_{(1)} \mathbf{A} \mathbf{P}_{(1)}^{\mathrm{T}}$ yields a matrix for which the $(i, j)$ element is $a_{\sigma(i) \sigma(j)}$, and that $\mathbf{P}_{(1)} \mathbf{P}_{(1)}^{\mathrm{T}}=\mathbf{P}_{(1)}^{\mathrm{T}} \mathbf{P}_{(1)}=\mathbf{I}$. That is, the similarity transformation $\mathbf{P}_{(1)} \mathbf{A} \mathbf{P}_{(1)}^{\mathrm{T}}$ gives a consistent renumbering of the rows and columns of $\mathbf{A}$ by the permutation $\sigma$.

The permutation $\sigma$ (or the permutation matrix $\mathbf{P}_{(1)}$ in (6.3)) constitutes a new coordinate system for analyzing the Jacobian matrix of the adjustment process. Under the new coordinate system, the SDM D can be represented as

$$
\mathbf{D}^{\times} \equiv \mathbf{P}_{(1)} \mathbf{D} \mathbf{P}_{(1)}^{\mathrm{T}}=\left[\begin{array}{c:c}
\mathbf{D}^{(0)} & \mathbf{D}^{(1)} \\
\hdashline \mathbf{D}^{(1) \mathrm{T}} & \mathbf{D}^{(0)}
\end{array}\right],
$$

where each of the submatrices $\mathbf{D}^{(0)}$ and $\mathbf{D}^{(1)}$ is a $M$-by- $M$ circulant generated from vectors $\quad \mathbf{d}_{0}^{(0)} \equiv\left[1, r^{2}, r^{4}, \ldots, r^{M}, \ldots, r^{4}, r^{2}\right]^{\mathrm{T}} \quad$ and $\quad \mathbf{d}_{0}^{(1)} \equiv\left[r, r^{3}, \ldots, r^{M-1}, r^{M-1}, \ldots, r^{3}, r\right]^{\mathrm{T}}$, respectively. Similarly, the Jacobian matrix $\nabla \mathbf{F}\left(\mathbf{h}^{*}\right)$ of the adjustment process is transformed into

$$
\nabla^{\times} \mathbf{F}\left(\mathbf{h}^{*}\right) \equiv \mathbf{P}_{(1)} \nabla \mathbf{F}\left(\mathbf{h}^{*}\right) \mathbf{P}_{(1)}^{\mathrm{T}}=H \mathbf{J}^{\times}\left(\mathbf{h}^{*}\right) \nabla^{\times} \mathbf{v}\left(\mathbf{h}^{*}\right)-\mathbf{I},
$$

where the Jacobian matrices in the right-hand side are, respectively, defined as

$$
\begin{aligned}
& \mathbf{J}^{\times}\left(\mathbf{h}^{*}\right) \equiv \mathbf{P}_{(1)} \mathbf{J}\left(\mathbf{h}^{*}\right) \mathbf{P}_{(1)}^{\mathrm{T}}=\left[\begin{array}{c:c}
\mathbf{J}^{(00)} & \mathbf{J}^{(01)} \\
\hdashline \mathbf{J}^{(10)} & \mathbf{J}^{(11)}
\end{array}\right], \\
& \nabla^{\times} \mathbf{v}\left(\mathbf{h}^{*}\right) \equiv \mathbf{P}_{(1)} \nabla \mathbf{v}\left(\mathbf{h}^{*}\right) \mathbf{P}_{(1)}^{\mathrm{T}}=\frac{1}{2 h}\left[\begin{array}{c:c}
\mathbf{V}^{(00)} & \mathbf{V}^{(01)} \\
\hdashline \mathbf{V}^{(10)} & \mathbf{V}^{(11)}
\end{array}\right] .
\end{aligned}
$$

For these Jacobian matrices under the new coordinate system, we have

Lemma 6.1.1: All of the submatrices $\mathbf{J}^{(i j)}$ and $\mathbf{V}^{(i j)}(i, j=0,1)$ are circulants. 
This leads to the following lemma, which significantly facilitates obtaining the eigenvalues $\mathbf{g}^{*}=\left\{g_{k}^{*}\right\}$ of the Jacobian matrix $\nabla \mathbf{F}\left(\mathbf{h}^{*}\right)$ :

Lemma 6.1.2: The eigenvalues $g_{k}^{*}(k=1,2, \ldots, K-1)$ of the Jacobian matrix $\nabla \mathbf{F}\left(\mathbf{h}^{*}\right)$ of the adjustment process at $\mathbf{h}^{*}=[2 h, 0,2 h, 0, \ldots, 2 h, 0]$ are represented as

$$
g_{k}^{*}=\left\{\begin{array}{ll}
\theta e_{k / 2}^{(00)}-1 & (k: \text { even }) \\
-1 & (k: \text { odd })
\end{array},\right.
$$

where $\mathbf{e}^{(00)} \equiv\left[e_{0}^{(00)}, e_{1}^{(00)}, \ldots, e_{M-1}^{(00)}\right]^{\mathrm{T}}$ denotes the eigenvalues of the Jacobian $\mathbf{V}^{(00)}$.

This lemma implies that knowing only $\mathbf{V}^{(00)}$ is sufficient to obtain the eigenvalues $\mathbf{g}^{*}$ of the Jacobian matrix $\nabla \mathbf{F}\left(\mathbf{h}^{*}\right)$. Furthermore, the submatrix $\mathbf{V}^{(00)}$ is a circulant consisting only of submatrices $\mathbf{D}^{(0)}, \mathbf{D}^{(1)}$, and $\mathbf{D}^{(1) \mathrm{T}}$ of the SDM D. Since they are circulants, for which DFT of vectors $\mathbf{d}_{0}{ }^{(0)}, \mathbf{d}_{0}{ }^{(1)}$, and $\mathbf{d}_{0}{ }^{(1 \mathrm{~T})}$ yields the associated eigenvalues $\mathbf{f}^{(0)}, \mathbf{f}^{(1)}$, and $\mathbf{f}^{(1 \mathrm{~T})}$, we can obtain the eigenvalues $\mathbf{e}^{(00)}$ of the matrix $\mathbf{V}^{(00)}$ :

Lemma 6.1.3: The Jacobian $\mathbf{V}^{(00)}$ and the associated eigenvalues $\mathbf{e}^{(00)}$ are given by

$$
\begin{aligned}
& \mathbf{V}^{(00)}=b\left[\mathbf{D}^{(0)} / d_{(0)}\right]-a_{1}\left\{(1+2 h)\left[\mathbf{D}^{(0)} / d_{(0)}\right]^{2}+\left[\mathbf{D}^{(1)} \mathbf{D}^{(1)}{ }^{\mathrm{T}}\right] / d_{(1)}^{2}\right\} \\
& \mathbf{e}^{(00)}=b\left[\mathbf{f}^{(0)} / d_{(0)}\right]-a_{1}\left\{(1+2 h)\left[\mathbf{f}^{(0)} / d_{(0)}\right]^{2}+\left[\mathbf{f}^{(1)}\right] \cdot\left[\mathbf{f}^{(1 \mathrm{~T})}\right] / d_{(1)}^{2}\right\}
\end{aligned}
$$

where $b \equiv(\sigma-1)^{-1}+\sigma^{-1}$ and $a_{1} \equiv \sigma^{-1}(2 h)^{-1} ; d_{(i)} \equiv \mathbf{d}_{0}^{(i)} \cdot \mathbf{1}(i=0,1)$.

Combining this with (6.7) in Lemma 6.1.2, we have

Proposition 6.1: The largest eigenvalue of the Jacobian matrix $\nabla \mathbf{F}\left(\mathbf{h}^{*}\right)$ of the adjustment process of (3.14) at $\mathbf{h}^{*}$ is given by

$$
\max _{k} \cdot\left\{g_{k}^{*}\right\}=g_{M}^{*}=\theta G^{*}\left(f_{M / 2}^{*}(r)\right) \text { for } \forall r \in(0,1],
$$

and the associated eigenvector is the $M \equiv K / 2$ th row vector $\mathbf{z}_{M}^{*}$ of $\mathbf{Z}^{*} \equiv \mathbf{P}_{(1)}^{\mathrm{T}} \mathbf{Z}^{\times} \mathbf{P}_{(1)}$, where $\mathbf{Z}^{\times} \equiv \operatorname{diag}\left[\mathbf{Z}_{[M]}, \mathbf{Z}_{[M]}\right], \mathbf{Z}_{[M]}$ is an M-by-M DFT matrix; $f_{M / 2}^{*}(r) \equiv c\left(r^{2}\right)^{2}$,

$$
\begin{aligned}
& G^{*}(x) \equiv b x-a^{*} x^{2}-\theta^{-1}, \\
& a^{*} \equiv a_{1}(1+2 h)=\sigma^{-1}\left(1+(2 h)^{-1}\right), \quad b \equiv(\sigma-1)^{-1}+\sigma^{-1} .
\end{aligned}
$$

This proposition allows us to determine the critical value at which a bifurcation from the $M$-centric pattern $\mathbf{h}^{*}=[2 h, 0,2 h, 0, \ldots, 2 h, 0]$ occurs. In a similar manner to the discussions for the first bifurcation from the uniform distribution $\overline{\mathbf{h}}$, we see that the second bifurcation from the $M$-centric pattern $\mathbf{h}^{*}$ occurs when the maximum 
eigenvalue $g_{M}^{*}(x)$ changes sign. Accordingly, the critical values of $f_{M / 2}^{*} \equiv c\left(r^{2}\right)^{2}$ at which the second bifurcation occurs are the solutions of a quadratic equation $G^{*}(x)=0$. For the homogeneous consumer case (i.e., $\theta \rightarrow+\infty$ ), the quadratic equation reduces to

$$
\lim _{\theta \rightarrow+\infty} G^{*}(x)=b x-a^{*} x^{2}=0,
$$

and hence, the critical values (i.e., the solutions of (6.10)) are given by

$$
x_{+}^{* *}=b / a^{*}=b \sigma /\left\{1+(2 h)^{-1}\right\} \text {, and } x_{-}^{* *}=0 .
$$

Note here that $f_{M / 2}^{*}$ is a monotonically increasing function of $\tau$. This implies that, in the course of decreasing $\tau$, the second bifurcation occurs when $\tau$ first reaches the critical value $\tau_{+}^{* *}$ that satisfies $x_{+}^{* *}=c\left(r\left(\tau_{+}^{* *}\right)^{2}\right)^{2}$. That is, the critical value of the second bifurcation in terms of the SDF is given by

$$
r\left(\tau_{+}^{* *}\right)=\left[\left(1-\left(x_{+}^{* *}\right)^{1 / 2}\right) /\left(1+\left(x_{+}^{* *}\right)^{1 / 2}\right)\right]^{1 / 2} .
$$

We can also identify the associated agglomeration pattern that emerges at this bifurcation. The direction of movement away from $\mathbf{h}^{*}$ at this bifurcation is the $M$ th eigenvector of $\mathbf{Z}^{*}: \mathbf{z}_{M}^{*}=[1,0,-1,0, \ldots, 1,0,-1,0]$. Accordingly, the pattern of agglomeration is given by

$$
\mathbf{h}=\mathbf{h}^{*}+\delta \mathbf{z}_{M}^{*}=[2 h+\delta, 0,2 h-\delta, 0, \ldots] \quad(0 \leq \delta \leq 2 h) .
$$

Thus, the properties of the second bifurcation can be summarized as follows:

Proposition 6.2: Suppose that the M-centric pattern $\mathbf{h}^{*}=[2 h, 0,2 h, 0, \ldots]$ is a stable equilibrium for the CP model with homogeneous consumers. With the decreases in $\tau$, the M-centric pattern $\mathbf{h}^{*}$ becomes unstable at the second break point $\tau=\tau_{+}^{* *}$ given by (6.11) and (6.12), and a more concentrated pattern $\mathbf{h}=[2 h+\delta, 0,2 h-\delta, 0, \ldots]$ $(0 \leq \delta \leq 2 h)$ then emerges.

After the second bifurcation, the deviation $\delta$ from the $M$-centric pattern $\mathbf{h}^{*}$ monotonically increases with the decrease in the transportation $\operatorname{cost} \tau$, which leads to an $(M / 2)$-centric pattern, $\mathbf{h}^{* *}=[4 h, 0,0,0, \ldots, 4 h, 0,0,0]$. That is, half of the core regions in the $M$-centric pattern $\mathbf{h}^{*}$ are completely taken over by the neighboring new core regions in $\mathbf{h}^{* *}$. This fact can be confirmed by examining a sustain point for the $(M / 2)$-centric pattern. As shown in Appendix 3, the equilibrium condition for $\mathbf{h}^{* *}$,

$$
v_{0}\left(\mathbf{h}^{* *}\right)=\max _{k}\left\{v_{k}\left(\mathbf{h}^{* *}\right)\right\},
$$

is satisfied for any $\tau$ smaller than some critical value. 


\subsection{Recursive Bifurcations - Spatial Period Doubling Bifurcations}

The analysis of the first and second bifurcations thus far reveals that the first and second bifurcations share a common property whereby the number of core regions is reduced by half and the spacing between each pair of neighboring core-regions doubles after each bifurcation. An intuitive explanation for this striking regularity is that the $K / 2$-centric pattern $\mathbf{h}^{*}$ in an RE with $K$ regions and a uniform pattern in an $\mathrm{RE}$ with $K / 2$ regions can be regarded as approximately equivalent. As shown in Lemma 6.1.2, the occurrence of the bifurcation from $\mathbf{h}^{*}$ is determined by only the marginal utilities $\mathbf{V}^{(00)}$ of skilled workers in core regions. Therefore, the bifurcation at $\mathbf{h}^{*}$ may be qualitatively similar to that of a uniform pattern in an RE with $K / 2$ regions, in which the $K / 4$-centric pattern should emerge at the first bifurcation. (For clarity of exposition, we assume below that the number of regions $K$ is a power of $2^{12}$.) Following this reasoning, we conjecture that, in the course of decreasing $\tau$, the multi-regional CP economy exhibits the spatial period doubling bifurcation (SPDB), whereby a third bifurcation at the $K /\left(2^{2}\right)$-centric pattern $\mathbf{h}^{* *}$ leads to a $K /\left(2^{3}\right)$ centric pattern $\mathbf{h}^{* * *}$ (in which alternate core regions of $\mathbf{h}^{* *}$ are extinct), at which a fourth bifurcation leads to a $K /\left(2^{4}\right)$-centric pattern, and such recursive bifurcations continue until a monocentric agglomeration is obtained (See Figure 5).

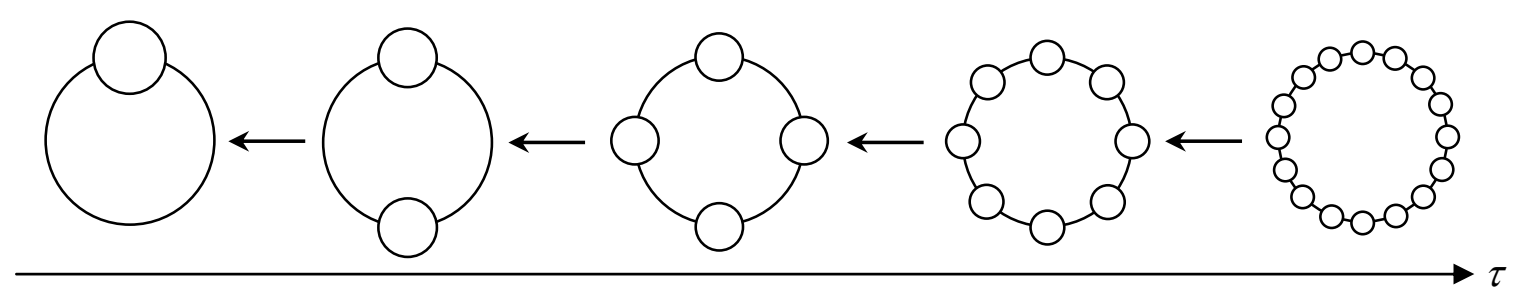

Figure 5. A series of agglomeration patterns that emerge in the course of decreasing $\tau$.

To a certain extent, the above conjecture is true, but the dispersion force due to unskilled workers living in periphery regions should not be overlooked. Since their import of M-sector goods affects the wages of skilled workers in the core regions, the marginal utilities $\mathbf{V}^{00}$ of the core regions in the $K$ regional system are not exactly the same as those in a $K / 2$ regional system. Thus, the occurrence of the SPDB is not necessarily obvious and must be proven.

In order to examine the possibility of the SPDB, we must obtain the eigenvalues $\mathbf{g}^{(n)}$ of the Jacobian matrix $\nabla \mathbf{F}\left(\mathbf{h}^{(n)}\right)$ at the $K / 2^{n}$-centric configuration,

$$
\mathbf{h}^{(n)} \equiv[\underbrace{2^{n} h, 0, \cdots 0}_{2^{n}}, \underbrace{2^{n} h, 0, \cdots 0}_{2^{n}}, \cdots, \underbrace{2^{n} h, 0, \cdots 0}_{2^{n}}]
$$

\footnotetext{
${ }^{12}$ The detailed discussion without this assumption is shown in Ikeda et al.(2009).
} 
which would emerge after the $n$th bifurcation from the flat earth equilibrium for an arbitrary natural number $n$. For this purpose, we introduce a particular division of the set of $C$ by which we exploit the symmetry of the configuration $\mathbf{h}^{(n)}$ so that the resultant submatrices of the Jacobian $\nabla \mathbf{F}\left(\mathbf{h}^{(n)}\right)$ reduce to circulants. First, recall that in obtaining the eigenvalues of $\nabla \mathbf{F}\left(\mathbf{h}^{*}\right)$ at a $K / 2$-centric configuration $\mathbf{h}^{*}$ that emerges after the first bifurcation, we have divided the set $C$ of regions into two subsets, namely, the subset $C_{0}=\{0,2, \ldots, K-2\}$ of regions with skilled workers and the subset $C_{1}=\{1,3, \ldots, K-1\}$ of regions without skilled workers. In a similar vein, for a $K / 4$-centric configuration $\mathbf{h}^{* *}$ that would emerge after the second bifurcation from the configuration $\mathbf{h}^{*}$, it is natural to divide each $C_{i}(i=0,1)$ into two subsets, $C_{i 0}$ and $C_{i 1}$, the elements of which are the even-numbered regions and the odd-numbered regions in $C_{i}$, respectively. It is also natural in the division to suppose that only the regions in subset $C_{00}$ have skilled workers in configuration $\mathbf{h}^{* *}$. This division procedure can be repeated recursively. For configuration $\mathbf{h}^{(n)}$ after $n$ bifurcations, each of the $2^{n-1}$ subsets $C_{i_{0} i_{1} \cdots i_{n-2}}\left(i_{0}, i_{1}, \cdots, i_{n-2}=0,1\right)$ of regions generated at the preceding $\left(n-1\right.$ th) bifurcation is divided into two subsets, $C_{i_{0} i_{1} \cdots i_{n-2} i_{n-1}}\left(i_{n-1}=0,1\right)$.

This division procedure naturally leads to the following definition of the permutation $\sigma_{K}^{n}$ of the set $C$ :

$$
\sigma_{K}^{n}:\left[\begin{array}{c:c:c:c:c}
0,1, \cdots, L-1 & L, \cdots, 2 L-1 & 2 L, \cdots, 3 L-1 & \cdots & K-L, \cdots, K-1 \\
C_{0 \cdots 000} & C_{0 \cdots 001} & C_{0 \cdots 010} & \cdots & C_{1 \cdots 111}
\end{array}\right],
$$

where $L \equiv K / 2^{n}$. Note here that the division and the associated permutation are the same as those used in the fast Fourier transform (FFT) algorithm (see, for example, Van Loan (1992)). This implies that the $k$ th element $\sigma_{K}^{n}(k)(k=0,1, \ldots, K-1)$ of the permutation $\sigma_{K}^{n}$ is given by a bit reversal operation used in the FFT technique whereby an index $k$, written in binary with digits $b_{K-1} b_{K-2} \cdots b_{1} b_{0}$, is transferred to the index with reversed digits $b_{0} b_{1} \cdots b_{K-2} b_{K-1}$, which yields $\sigma_{K}^{n}(k)$. It also follows that the first component of the subset $C_{i_{0} i_{1} \cdots i_{n-1}}$ is given by the bit reversal of $i_{0} i_{1} i_{2} \cdots i_{n-1}: a(n) \equiv i_{0}+i_{1} \cdot 2+\cdots+i_{n-1} 2^{n-1}$ (for a concrete illustration of the division of the set $C$ and the associated permutation $\sigma_{K}^{n}$, see Appendix 6).

The permutation $\sigma_{K}^{n}$ defined above can be expressed in terms of the associated $K \times K$ permutation matrix $\mathbf{P}_{(n)}$ :

$$
\mathbf{P}_{(n)} \equiv\left[\begin{array}{ccc}
\hat{\mathbf{p}}_{(n)} & & \mathbf{0} \\
& \ddots & \\
\mathbf{0} & & \hat{\mathbf{p}}_{(n)}
\end{array}\right] \mathbf{P}_{(n-1)}, \quad \hat{\mathbf{P}}_{(n)} \equiv\left[\begin{array}{c}
\mathbf{P}_{(n)}^{e} \\
\hdashline \mathbf{P}_{(n)}^{o}
\end{array}\right],
$$

where $\mathbf{P}_{(n)}^{e}$ and $\mathbf{P}_{(n)}^{o}$ are $K / 2^{n+1}$ by $K / 2^{n}$ matrices. The $(i, j)$ element of $\mathbf{P}_{(n)}^{e}$ is 1 if 
$j=2 i \quad\left(i=0,1,2, \ldots, K / 2^{n+1}-1\right)$, and is otherwise 0 , whereas that of $\mathbf{P}_{(n)}^{o}$ is 1 if $j=2 i+1$, and is otherwise 0 . Under the new coordinate system transformed by the permutation $\sigma_{K}^{n}$, the SDM D can be represented as

$$
\mathbf{D}_{(n)}^{\times} \equiv \mathbf{P}_{(n)} \mathbf{D P}_{(n)}^{\mathrm{T}} .
$$

Clearly, the transformed SDM, $\mathbf{D}_{(n)}^{\times}$, consists of $\left(2^{n}\right)^{2}$ blocks (submatrices), in which each of the $2^{n}$ block divisions in each of the row and column directions corresponds to the subset $C_{i_{0} i_{1} i_{2} \cdots i_{n-1}}\left(i_{0}, i_{1}, \cdots, i_{n-1}=0,1\right)$ of $C$. As it turns out, we do not have to deal with all of the submatrices. For our purpose, it is sufficient to focus on $2^{n}$ submatrices of the first block-row, in which each submatrix, denoted as $\mathbf{D}^{\left(i_{0} i_{1} i_{2} \cdots i_{n-1}\right)}$, represents the spatial discounting factors between the core regions of set $C_{(000 \cdots 0)}$ and the periphery regions of set $C_{i_{0} i_{1} i_{2} \cdots i_{n-1}}$. It is readily shown that the submatrix $\mathbf{D}^{\left(i_{0} i_{1} i_{2} \cdots i_{n-1}\right)}$ is a circulant generated from a vector $\mathbf{d}_{0}^{\left(i_{0} i_{1} i_{2} \cdots i_{n-1}\right)} \equiv \mathbf{d}_{0}^{(a(n))}$ with $L(n) \equiv K / 2^{n}$ elements, the $k$ th element $d_{0, k}^{(a(n))}$ of which is given by

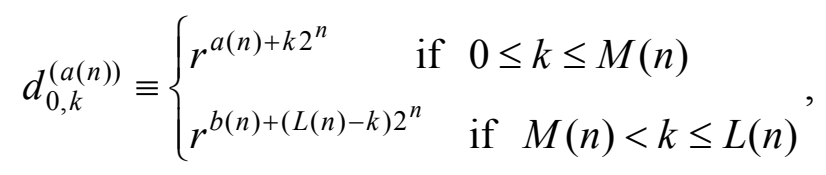

where $\quad a(n) \equiv i_{0}+i_{1} \cdot 2+\cdots+i_{n-1} 2^{n-1} \quad$ (i.e., the bit reversal of $i_{0} i_{1} i_{2} \cdots i_{n-1}(\bmod 2)=$ $\left.i_{n-1}+i_{n-2} \cdot 2+\cdots+i_{0} 2^{n-1}\right), b(n) \equiv 2^{n}-a(n)$, and $M(n) \equiv L(n) / 2$.

Similarly, applying this transformation to the Jacobian matrix $\nabla \mathbf{F}\left(\mathbf{h}^{*}\right)$ of the adjustment process, we have

$$
\nabla^{\times} \mathbf{F}\left(\mathbf{h}^{(n)}\right) \equiv \mathbf{P}_{(n)} \nabla \mathbf{F}\left(\mathbf{h}^{(n)}\right) \mathbf{P}_{(n)}^{\mathrm{T}}=H \mathbf{J}^{\times}\left(\mathbf{h}^{(n)}\right) \nabla^{\times} \mathbf{v}\left(\mathbf{h}^{(n)}\right)-\mathbf{I},
$$

where the Jacobian matrices in the right-hand side are, respectively, defined as

$$
\mathbf{J}^{\times}\left(\mathbf{h}^{(n)}\right) \equiv \mathbf{P}_{(n)} \mathbf{J}\left(\mathbf{h}^{(n)}\right) \mathbf{P}_{(n)}^{\mathrm{T}}, \quad \nabla^{\times} \mathbf{v}\left(\mathbf{h}^{(n)}\right) \equiv \mathbf{P}_{(n)} \nabla \mathbf{v}\left(\mathbf{h}^{(n)}\right) \mathbf{P}_{(n)}^{\mathrm{T}} .
$$

Each of the Jacobian matrices $\mathbf{J}^{\times}\left(\mathbf{h}^{(n)}\right)$ and $\nabla^{\times} \mathbf{v}\left(\mathbf{h}^{(n)}\right)$ has a block structure with $\left(2^{n}\right)^{2}$ submatrices, just like the transformed SDM. These submatrices under the new coordinate system have the following desirable properties:

Lemma 6.2.1: The submatrices $\mathbf{J}^{(0, a(n))}$ of $\mathbf{J}^{\times}\left(\mathbf{h}^{(n)}\right)$ and $\mathbf{V}^{(0, a(n))}$ of $\nabla^{\times} \mathbf{v}\left(\mathbf{h}^{(n)}\right),(a(n)$ $\left.\equiv i_{0}+i_{1} \cdot 2+\cdots+i_{n-1} 2^{n-1}=0,1,2, \ldots, 2^{n}-1\right)$, are circulants, where the superscript $(0$, $a(n))$ of each submatrix denotes that the block-row and the block-column of the submatrix correspond to set $C_{(000 \cdots 0)}$ and set $C_{i_{0} i_{1} i_{2} \cdots i_{n-1}}$, respectively.

This leads to the following result (parallel to Lemma 6.1.2 in the second bifurcation), which is very useful for obtaining the eigenvalues $\mathbf{g}^{(n)}=\left\{g_{k}^{(n)}\right\}$ of $\nabla \mathbf{F}\left(\mathbf{h}^{(n)}\right)$ : 
Lemma 6.2.2: The eigenvalues $g_{k}^{(n)}(k=0,1,2, \ldots, K-1)$ of the Jacobian matrix $\nabla \mathbf{F}\left(\mathbf{h}^{(n)}\right)$ of the adjustment process at $\mathbf{h}^{(n)}$ are represented as

$$
g_{k}^{(n)}= \begin{cases}\theta e_{k / 2^{n}}^{(0,0)}-1 & \left(k \bmod 2^{n}=0\right) \\ -1 & \text { (otherwise) }\end{cases}
$$

where $\mathbf{e}^{(0,0)} \equiv\left[e_{0}^{(0,0)}, e_{1}^{(0,0)}, \ldots, e_{L(n)-1}^{(0,0)}\right]^{\mathrm{T}}$ are the eigenvalues of the submatrix $\mathbf{V}^{(0,0)}$.

This lemma indicates that the eigenvalues $\mathbf{e}^{(0,0)}$ of the submatrix $\mathbf{V}^{(0,0)}$ immediately yield the eigenvalues $\mathbf{g}^{(n)}$. Since the $\mathbf{V}^{(0,0)}$ is a circulant consisting only of the submatrices $\mathbf{D}^{(a(n))} \equiv \mathbf{D}^{\left(i_{0} i_{1} i_{2} \cdots i_{n-1}\right)}$ of the SDM, we can obtain the eigenvalues $\mathbf{e}^{(0,0)}$ :

Lemma 6.2.3: The Jacobian $\mathbf{V}^{(0,0)}$ and the associated eigenvalues $\mathbf{e}^{(0,0)}$ are given by

$$
\begin{aligned}
& \mathbf{V}^{(0,0)}=b\left[\mathbf{D}^{(0)} / d_{(0)}\right]-a_{n}\left\{\left(1+2^{n} h\right)\left[\mathbf{D}^{(0)} / d_{(0)}\right]^{2}+\sum_{a(n)=1}^{2^{n}-1} \mathbf{D}^{(a(n))} \mathbf{D}^{(a(n)) \mathrm{T}} / d_{(a(n)))}^{2}\right\} \\
& \mathbf{e}^{(0,0)}=b\left[\mathbf{f}^{(0)} / d_{(0)}\right]-a_{n}\left\{\left(1+2^{n} h\right)\left[\mathbf{f}^{(0)} / d_{(0)}\right]^{2}+\sum_{a(n)=1}^{2^{n}-1}\left[\mathbf{f}^{(a(n))}\right] \cdot\left[\mathbf{f}^{(a(n) \mathrm{T})}\right] / d_{(a(n))}^{2}\right\},
\end{aligned}
$$

where $a_{n} \equiv \sigma^{-1}\left(2^{n} h\right)^{-1}, d_{(a(n))} \equiv \mathbf{d}_{0}^{(a(n))} \cdot \mathbf{1}, \mathbf{f}^{(a(n))}=\mathbf{Z}_{[L(n)]} \mathbf{d}_{0}^{(a(n))}, \mathbf{f}^{(a(n) \mathrm{T})}=\mathbf{Z}_{[L(n)]} \mathbf{d}_{0}^{(a(n) \mathrm{T})}$.

Straightforward calculation of (6.20b) together with Lemma 6.2.2 leads to the following proposition, which provides the key information by which to examine the occurrence of the $n+1$ th bifurcation from the $K / 2^{n}$-centric agglomeration pattern $\mathbf{h}^{(n)}$ :

Proposition 6.3: The largest eigenvalue of the Jacobian matrix $\nabla \mathbf{F}\left(\mathbf{h}^{(n)}\right)$ of the adjustment process of (3.14) at $\mathbf{h}^{(n)}$ is given by

$$
\max _{k} .\left\{g_{k}^{(n)}\right\}=g_{M}^{(n)}=\theta G^{(n)}(r) \quad \text { for } \forall r \in(0,1],
$$

and the associated eigenvector is the $M$ th row vector $\mathbf{z}_{M}^{(n)}$ of $\mathbf{Z}_{(n)} \equiv \mathbf{P}_{(n)}^{\mathrm{T}} \mathbf{Z}_{(n)}^{\times} \mathbf{P}_{(n)}$, where $\mathbf{Z}_{(n)}^{\times} \equiv \operatorname{diag}\left[\mathbf{Z}_{[L(n)]}, \cdots, \mathbf{Z}_{[L(n)]}\right], \mathbf{Z}_{[L(n)]}$ is an $L(n)$-by-L(n) DFT matrix, where $L(n) \equiv K / 2^{n}$,

$$
\begin{aligned}
& G^{(n)}(r) \equiv c_{0}(r, n) \cdot \hat{G}^{(n)}(r)-\theta^{-1}, \\
& \hat{G}^{(n)}(r) \equiv b-a_{n}\left\{\left(1+2^{n} h\right) c_{0}(r, n)+\sum_{j=1}^{2^{n}-1} c_{j}(r, n)\right\} \\
& c_{j}(r, n) \equiv\left[\left(1-r^{2^{n}-2 j}\right) /\left(1+r^{2^{n}-2 j}\right)\right]^{p(j, n, K)} \quad\left(j=0,1,2, \ldots, 2^{n}-1\right),
\end{aligned}
$$$$
p(j, n, K)=\left\{\begin{array}{ll}
1 & \text { if } j=0 \text { and } M(n) \equiv L(n) / 2=1 \\
2 & \text { otherwise }
\end{array} .\right.
$$ 
The $n+1$ th bifurcation for the homogeneous consumer case (i.e., $\theta \rightarrow+\infty$ ) occurs at the critical value $r_{(n)}$ of the SDF at which the largest eigenvalue $g_{M}^{(n)}$ changes sign, that is, $r^{(n)}$ is the solution of

$$
\lim _{\theta \rightarrow+\infty} G^{(n)}(r)=0 \quad\left(\Leftrightarrow \hat{G}^{(n)}(r)=0\right) .
$$

In order for the SPDB to occur in the course of decreasing $\tau$ (i.e., increasing $r$ ), the critical value $r^{(n)}$ must be an increasing function of $n$ :

$$
r^{(n)}<r^{(n+1)} \quad \text { for } n=1,2, \ldots, \log _{2} K-1 .
$$

The following lemma helps to determine whether the condition of (6.24) holds:

Lemma 6.2.4: The function $\hat{G}^{(n)}(r)$ of the $S D F r$ has the following properties:

(i) $\hat{G}^{(n)}(r)$ is a monotonically increasing function of $r \in[0,1]$.

(ii) $\hat{G}^{(n)}(0)=b-\sigma^{-1}\left\{1+h^{-1}-\left(2^{n} h\right)^{-1}\right\}, \quad \hat{G}^{(n)}(1)=b>0$.

(iii) For any $n \geq 1, \hat{G}^{(n)}(r) \geq \hat{G}^{(n+1)}(r) \quad \forall r \in[0,1]$. (The equality holds only if $r=1$.)

From properties (i) and (ii) of $\hat{G}^{(n)}(r)$ in Lemma 6.2.5, (6.23) has a unique solution $r_{(n)} \in[0,1]$ if and only if $\hat{G}^{(n)}(0)<0$, which is equivalently written as

$$
h<\left(1+\sigma^{-1}\right)\left(1-\left(2^{n}\right)^{-1}\right) \text {. }
$$

Furthermore, if this condition holds, then it can be concluded from property (iii) of $\hat{G}^{(n)}(r)$ that the inequality of (6.24) holds, i.e., the SPDB occurs in the CP model. Note that the condition of (6.25) for the occurrence of the SPDB is very similar to the no-black-hole condition (for the homogeneous consumer case), $h<\left(1+\sigma^{-1}\right)$, obtained in Proposition 5.1. The right-hand side of (6.25) increases with $n$ (i.e., the requirement in (6.25) becomes "weak" with the increases of $n$ ), and the condition of (6.25) reduces to the no-black-hole condition as $n$ becomes large. Therefore, we can predict that, if the no-black-hole condition is satisfied and the first bifurcation occurs, then the succeeding SPDB necessarily occurs in the CP model.

Finally, it is easily verified that the associated agglomeration pattern that emerges at the $n+1$ th bifurcation is consistent with the SPDB. The direction of movement away from $\mathbf{h}^{(n)}$ at this bifurcation is the $M \equiv K / 2$ th eigenvector $\mathbf{z}_{M}^{(n)}$ of $\mathbf{Z}_{(n)}$. Accordingly, the pattern of agglomeration is given by

$$
\begin{aligned}
& \mathbf{h}=\mathbf{h}^{(n)}+\delta \mathbf{z}_{M}^{(n)} \quad\left(0 \leq \delta \leq 2^{n} h\right), \\
& \mathbf{z}_{M}^{(n)}=[\underbrace{1,0, \cdots 0}_{2^{n}}, \underbrace{-1,0, \cdots 0}_{2^{n}}, \cdots \cdots, \underbrace{1,0, \cdots 0}_{2^{n}}, \underbrace{-1,0, \cdots 0}_{2^{n}}]
\end{aligned}
$$

which means that alternate halves of $K / 2^{n}$ core regions in $\mathbf{h}^{(n)}$ absorb skilled 
workers from the neighboring core regions. Further decreases in the transportation cost $\tau$ increase the deviation $\delta$ from the $K / 2^{n}$-centric pattern $\mathbf{h}^{(n)}$ and eventually lead to a $K / 2^{n+1}$-centric agglomeration pattern $\mathbf{h}^{(n+1)}$. This is consistent with the SPDB rule. Thus, we can confirm the occurrence of the SPDB in the CP model:

Proposition 6.4: Consider the bifurcation process of the CP model with homogeneous consumers in the RE with $K$ regions in the course of decreasing transportation cost $\tau$.

(i) The $K / 2^{n}$-centric pattern $\mathbf{h}^{(n)}$ becomes unstable at the $n+1$ th break point (in terms of the $S D F$ ) $r=r^{(n)}$ given by the solution of (6.23).

(ii) If the CP model satisfies (6.25), then the CP model exhibits a series of spatial period doubling bifurcations, in which the number of core regions is reduced by half and the spacing between each pair of neighboring core-regions doubles after each bifurcation, and the recursive bifurcations continue until a mono-centric agglomeration is reached.

\subsection{Numerical Examples}

We present a number of numerical examples to illustrate the theoretical results. We show the cases of (i) homogeneous consumers and (ii) heterogeneous consumers. In (ii), we demonstrate two examples, (ii-1) and (ii-2), which correspond to the cases with single re-dispersion and multiple re-dispersions, respectively. For the computation of these examples, we use the algorithm developed by Ikeda et al. (2009), which applies the computational bifurcation theory to the multi-regional CP model. This algorithm allows us to obtain a complete picture of the evolutionary process of the agglomeration patterns, which includes not only the theoretical results but also transition processes of (i) and agglomeration patterns of (ii). The results of the numerical analysis are shown in Figure 6, in which the horizontal axis denotes the SDF $r$ and the curve represents the fraction $h_{i} / H$ of the skilled workers in the most populous region.

$\underline{\text { Case (i) }}$ The parameter values in this case are $\sigma=2.1, \theta=3000, H=1.6$, and $K=16$ ( $\theta$ is sufficiently large, so that the equilibrium configurations can be regarded as those of the homogeneous consumer case). The evolutionary process of the agglomeration pattern in the course of increasing $r$ is illustrated in Figure 6(a). This figure shows the occurrence of the SPDB. Specifically, the recursive bifurcations lead, in turn, to the 8-centric, 4-centric, duocentric and monocentric patterns. Furthermore, it is confirmed that each break point $r^{(n)}$ derived from the numerical analysis coincides with the theoretical prediction (i.e., the solution of (6.23)). These results are consistent with Proposition 6.4. 


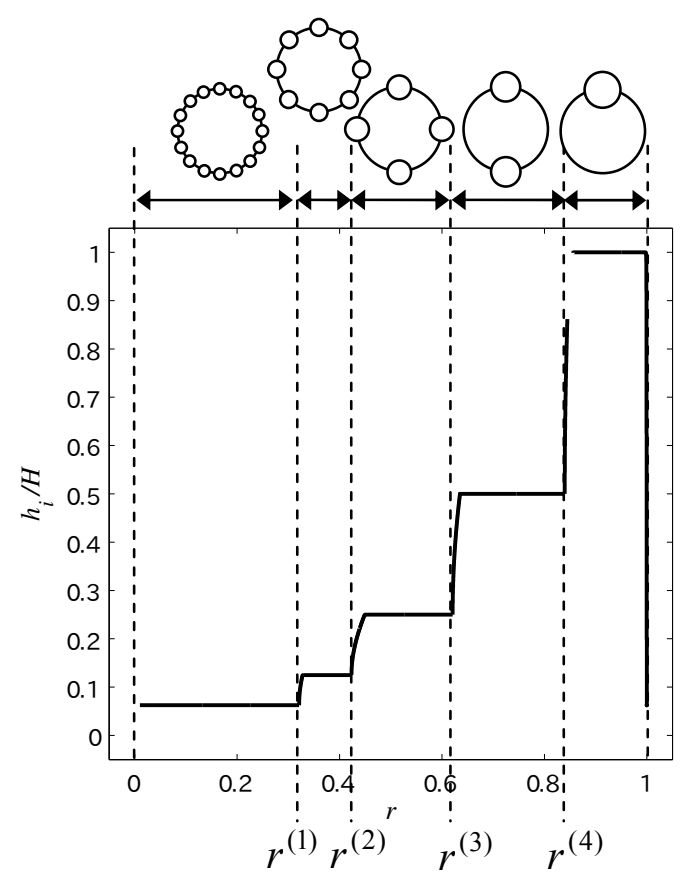

(a) Case (i): Homogeneous consumer

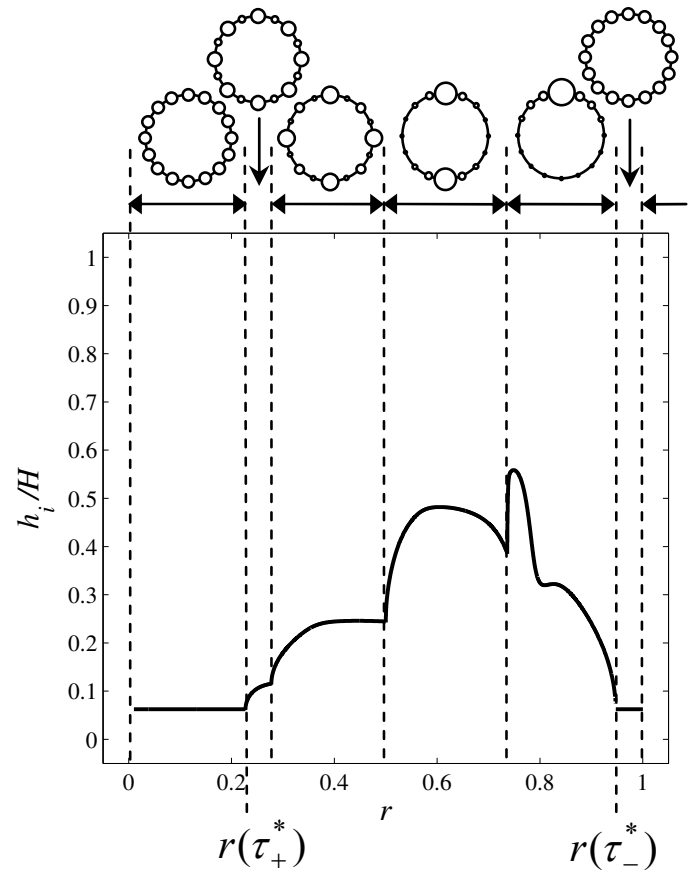

(b) Case (ii-1): Heterogeneous consumer

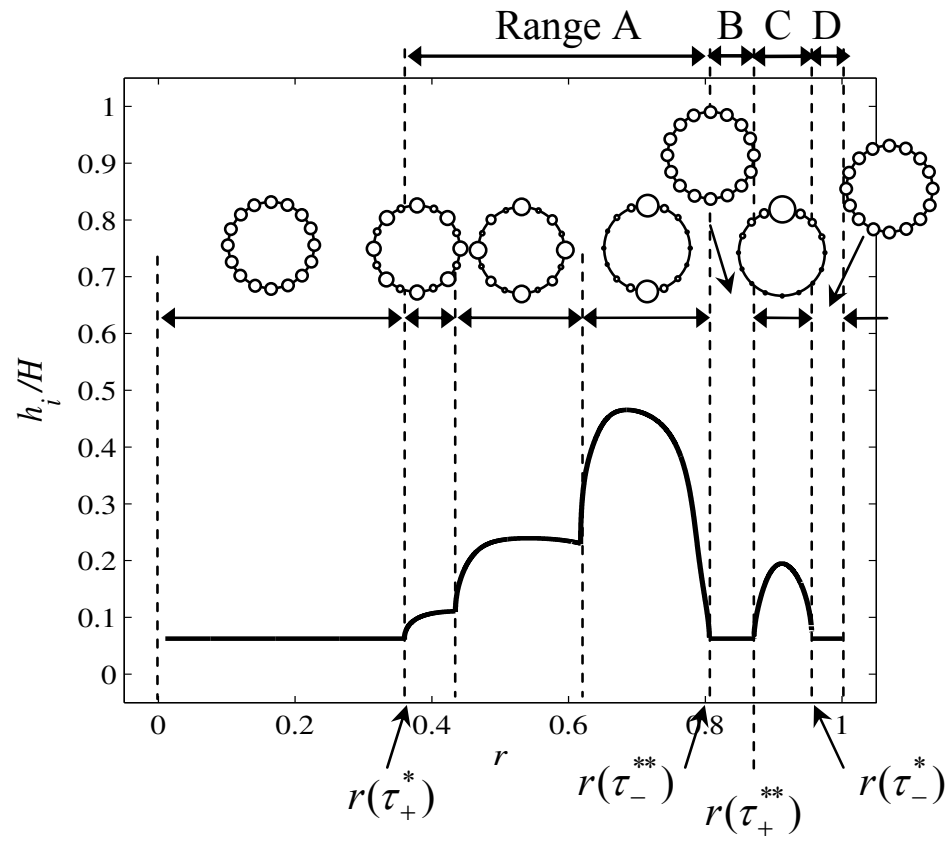

(c) Case (ii-2): Heterogeneous consumer case with multiple re-dispersions

Figure 6. Evolution of the agglomeration patterns

Case (ii-1) The parameter values are $\sigma=2.1, \theta=10, H=3.2$, and $K=16$. The evolutionary process of this case, which is illustrated in Figure $6(b)$, is qualitatively the same as that of the case (i). The agglomeration patterns of both cases evolve from a uniform distribution toward the 8-centric, 4-centric, duocentric, and monocentric patterns in turn as $r$ increases. However, there are two main differences between this 
and case (i). As shown in Proposition 5.3 (iii), the re-dispersion from the monocentric pattern occurs at $r\left(\tau_{-}^{*}\right)$, and the population of any region never becomes zero.

Case (ii-2) The parameter values are identical to those of case (ii-1), except for $\sigma=2.5$. As illustrated in Figure 6(c), multiple re-dispersions occur in the course of increasing $r$. The first re-dispersion is observed at the boundary $r\left(\tau_{-}^{* *}\right)$ between range $\mathrm{A}$ and $\mathrm{B}$. That is, unlike case (ii-1), the increase in $r$ from range $\mathrm{A}$ to range $\mathrm{B}$ causes the collapse of the duocentric agglomeration, which leads to the uniform distribution. After the emergence of a monocentric pattern in range $\mathrm{C}$, the second re-dispersion occurs at the boundary $r\left(\tau_{-}^{*}\right)$ between range $\mathrm{C}$ and range $\mathrm{D}$. These results are consistent with Proposition 5.3 (iii).

\section{Concluding Remarks}

We have presented an approach to capture the bifurcation properties of the multiregional core-periphery $(\mathrm{CP})$ model. The presented method is characterized by three key tools: a spatial discounting matrix (SDM), discrete Fourier transformation (DFT), and a discrete racetrack economy (RE). An effective combination of these tools overcomes the limitations of the Turing (1952) approach, enabling an examination of whether agglomeration of mobile factors emerges from a uniform distribution and allowing the evolution of spatial agglomeration patterns exhibited by the CP model when the transportation cost steadily decreases to be traced analytically. Through the analysis, we revealed a number of interesting behaviors of the CP model, and notably, we proved analytically the occurrence of spatial period doubling bifurcation, in which the number of core regions reduces by half and the spacing between each pair of neighboring core-regions doubles after each bifurcation, and the recursive bifurcations continue until a mono-centric agglomeration is reached.

Although we restricted ourselves to the analysis of the CP model, the approach presented herein can be used for a wide class of models involving spatial agglomeration of economic activities. For instance, analyzing the other variants of the CP model, such as that by Ottaviano et al. (2002), is straightforward. Note that the application area of the proposed approach is not restricted to inter-regional migration models but also covers the analysis of a variety of urban land-use (internal structure of cities) models, in which the formation of urban centers is endogenized (see, for example, Fujita and Thisse (2002, Chapters 6 and 7)). A typical example of such an application can be found in Akamatsu and Takayama (2009b).

In order to deal with agglomeration models having more complex structure/ 
circumstances, the proposed method may be extended in several ways. A straightforward extension would be to apply the method in two-dimensional space. This may be achieved in a relatively straightforward manner by replacing the onedimensional DFT in the present method with the two-dimensional DFT. Another interesting extension would be to allow for multiple types of mobile agents. There are a number of spatial economic models of this type. Examples include NEG models with multiple industry sectors, models of the emergence of new cities (Fujita and Mori (1997)), and self-organizing urban structure models in which the spatial interactions between mobile firms and consumers result in the formation of CBDs (e.g., Fujita and Ogawa (1982), Fujita (1988), Berliant et al.(2002), Lucas and RossiHansberg (2002), Berliant and Wang (2008)). This extension would be challenging, but would greatly enhance our understanding of the nature of agglomeration economies.

\section{Acknowledgments}

We are grateful for comments and suggestions made by Masahisa Fujita, Tomoya Mori, Se-il Mun, Yasusada Murata, Toshimori Otazawa, Daisuke Oyama, Yasuhiro Sato, Takatoshi Tabuchi, Kazuhiro Yamamoto, Dao-Zhi Zeng, as well as participants at the 23th Applied Regional Science Conference Meeting in Yamagata. Funding from the Japan Society for the Promotion of Science, Grant-in-Aid for Scientific Research (B) 19360227/21360194 is greatly acknowledged.

\section{Appendix 1: Proofs of Lemma 4.1 and Proposition 4.1}

Proof of Lemma 4.1: A calculation of (4.5) yields that

$$
f_{m}=1+(-1)^{m} r^{M}+\sum_{k=1}^{M-1}\left(w^{m k}+w^{-m k}\right) r^{k}=\frac{\left(1-r^{2}\right)\left(1-(-1)^{m} r^{M}\right)}{1-r\left(w^{m}+w^{-m}\right)+r^{2}}
$$

$(m=0,1, \ldots, M)$.

(A1.1b) $\quad f_{m}=f_{K-m} \quad(m=1,2, \ldots, M-1)$.

Substituting $w \equiv \exp [\mathrm{i}(2 \pi / K)]$ into (A1.1a), we obtain (4.6a). QED

Proof of Proposition 4.1:

(i) Since $\mathbf{D}$ is diagonalized by the similarity transformation shown in (4.4), the k-th eigenvector is given by (4.7) in Proposition 4.1.

(ii) Using $\overline{\mathbf{f}}=\mathbf{Z} \mathbf{d}_{0} / d$ and Lemma 4.1, we can obtain (4.8).

(iii) If $m$ is even, differentiating $\bar{f}_{m}$ with respect to $r$ yields

$$
\frac{d \bar{f}_{m}}{d r}=-\frac{2\left(1-r^{2}\right)\left(1-\cos _{m}\right)}{\left(1-2 r \cos _{m}+r^{2}\right)^{2}},
$$

where $\cos _{m} \equiv \cos (2 \pi m / K)$. Since $-1 \leq \cos _{m} \leq 1, d \bar{f}_{m} / d r<0$. If $m$ is odd, we can rewrite the 
condition $d \bar{f}_{m} / d r<0$ as

$$
\cos _{m}<1+\frac{M r^{M}(2-y)}{x-2 M r^{M}(1-r)^{2}}
$$

where $x \equiv\left(1-r^{2}\right)\left(1-r^{2 M}\right)>0, y \equiv r(1-r)^{2}\left(1+r^{2}\right)<2$. Since the second term of the right-hand side of (A1.2) is positive, this inequality is always satisfied. Therefore, $\bar{f}_{m}$ is a monotonically decreasing function of $r$ for $\forall r \in[0,1)$.

(iv) Since $c_{m}(r)$ is a increasing function of $\cos _{m}, \bar{f}_{m}$ is also a increasing function of $\cos _{m}$. Thus, we obtain $\max _{m}\left\{\bar{f}_{m}\right\}=\bar{f}_{0}, \min _{m}\left\{\bar{f}_{m}\right\}=\bar{f}_{M}$.

(v) From the facts that $\bar{f}_{m}$ is a increasing function of $\cos _{m}, \bar{f}_{1} \leq 1$ and $\bar{f}_{M-1}>c_{M}^{2} \varepsilon_{M}$, we obtain the inequalities in (v). QED

\section{Appendix 2: Proofs of Lemmas 4.2 and 4.3}

Proof of Lemma 4.2: From the definition of $\mathbf{M}, \mathbf{M} \equiv \mathbf{D} \Delta^{-1}$, we can reduce $\mathbf{M}$ at $\overline{\mathbf{h}}$ to $\mathbf{M}(\overline{\mathbf{h}})=(h d)^{-1} \mathbf{D}$. Substituting this into (3.19) yields (4.11). Substituting $\mathbf{h}=\overline{\mathbf{h}}$ into (3.18), we obtain (4.10).

Proof of Lemma 4.3: We examine each of $\nabla \mathbf{v}(\overline{\mathbf{h}})$ and $\mathbf{J}(\overline{\mathbf{h}})$ in turn. The right-hand side of (4.11) consists only of additions and multiplications of the circulant matrix $\mathbf{D}$. It follows from this that $\nabla \mathbf{v}(\overline{\mathbf{h}})$ is a circulant. (4.10) clearly show that $\mathbf{J}(\overline{\mathbf{h}})$ is a circulant because $\mathbf{I}$ and $\mathbf{1 1}^{\mathrm{T}}$ in the righthand side are obviously circulants. Thus, both $\nabla \mathbf{v}(\overline{\mathbf{h}})$ and $\mathbf{J}(\overline{\mathbf{h}})$ are circulants, and this concludes that the Jacobian matrix $\nabla \mathbf{F}(\overline{\mathbf{h}})$ at the configuration $\overline{\mathbf{h}}$ is a circulant.

\section{Appendix 3: Sustain points for $h^{*}$ and $h^{* *}$}

We will show the derivation of sustain points for $\mathbf{h}^{*}$ and $\mathbf{h}^{* *}$, in turn.

(1) For the $M$-centric pattern $\mathbf{h}^{*}=[2 h, 0,2 h, 0, \cdots, 2 h, 0]$, we can easily obtain the indirect utility for each city by substituting $\mathbf{h}=\mathbf{h}^{*}$ into (3.12):

$$
v_{i}\left(\mathbf{h}^{*}\right)=\left\{\begin{array}{lc}
\sigma^{-1}\left[1+h^{-1}\right]+(\sigma-1)^{-1} \ln \left(2 h d_{(0)}\right) & (i \bmod 2=0) \\
\sigma^{-1}\left[(2 h)^{-1}\left(x^{-1}+(1+2 h) x\right)\right]+(\sigma-1)^{-1} \ln \left(2 h d_{(1)}\right) & \text { (otherwise) }
\end{array}\right.
$$

where $x \equiv d_{(1)} / d_{(0)}, d_{(i)} \equiv \mathbf{d}_{0}^{(i)} \cdot \mathbf{1}$. To obtain the sustain point for $\mathbf{h}^{*}$, we represent the utility difference between the "core" cities and the "periphery" cities as a function of the SDF:

$$
v_{01}(r) \equiv v_{0}\left(\mathbf{h}^{*}\right)-v_{1}\left(\mathbf{h}^{*}\right)=-(1+2 h) x^{2}+2(1+h) x-1-2 h \sigma(\sigma-1)^{-1}(x \ln x)
$$

Differentiating $v_{01}(r)$ with respect to $r$, we have

$$
\begin{aligned}
& \frac{d v_{01}(r)}{d r}=\frac{d x}{d r} \alpha(r), \\
& \alpha(r) \equiv-2(1+2 h) x-\frac{2 h \sigma}{\sigma-1} \ln x+2-\frac{2 h}{\sigma-1}, \\
& \frac{d x}{d r}=\frac{2\left(1-r^{2}\right)}{\left(1+r^{2}\right)^{2}}>0 .
\end{aligned}
$$

It follows from (A3.1) and (A3.2) that $\alpha(r)$ is a monotonically decreasing function of $r, \alpha(0)>0$ and $\alpha(1)<0$. This implies that $d v_{01}(r) / d r$ changes sign only once. That is, $v_{01}(r)$ is a unimodal 
function of $r$. From this fact, we see that $v_{01}(r)$ takes zero value at $r=1$ and $r=r_{01}^{*}>0$ (i.e., the equation $v_{01}(r)=0$ has two positive solutions 1 and $\left.r_{01}^{*}\right)$ and that

$$
v_{01}(r)\left\{\begin{array}{l}
<0 \text { for } 0<r<r_{01}^{*} \\
\geq 0 \text { for } r_{01}^{*} \leq r<1
\end{array} .\right.
$$

This means that the equilibrium condition for $\mathbf{h}^{*}, v_{0}\left(\mathbf{h}^{*}\right)=v_{2}\left(\mathbf{h}^{*}\right)=\max _{k}\left\{v_{k}\left(\mathbf{h}^{*}\right)\right\}$, is satisfied for any $r$ larger than $r_{01}^{*}$; that is, $r=r_{01}^{*}$ is the sustain point for $\mathbf{h}^{*}=[2 h, 0,2 h, 0, \cdots, 2 h, 0]$.

(2) The sustain point for the $M / 2$-centric pattern $\mathbf{h}^{* *}=[4 h, 0,0,0, \cdots, 4 h, 0,0,0]$ can be obtained by the similar manner. The indirect utility at $\mathbf{h}^{* *}$ is given by

$$
v_{i}\left(\mathbf{h}^{* *}\right)=\left\{\begin{array}{lc}
\sigma^{-1}\left[1+h^{-1}\right]+(\sigma-1)^{-1} \ln \left(4 h d_{(0)}\right) & \left(i \bmod 2^{2}=0\right) \\
\sigma^{-1}\left[(4 h)^{-1}\left(x_{01}+(1+4 h) x_{10}+x_{12}+x_{21}\right)\right]+(\sigma-1)^{-1} \ln \left(4 h d_{(1)}\right) & \left(i \bmod 2^{2}=1,3\right) \\
\sigma^{-1}\left[(4 h)^{-1}\left(2+x_{02}+(1+4 h) x_{20}\right)\right]+(\sigma-1)^{-1} \ln \left(4 h d_{(2)}\right) & \left(i \bmod 2^{2}=2\right)
\end{array}\right.
$$

where $x_{i j} \equiv d_{(i)} / d_{(j)}$. Define the following utility difference functions at $\mathbf{h}^{* *}$ :

$$
\begin{aligned}
& v_{01}(r) \equiv v_{0}\left(\mathbf{h}^{* * *}\right)-v_{1}\left(\mathbf{h}^{* * *}\right) \\
& =-(4 h+1)\left(x_{10}\right)^{2}+4(1+h) x_{10}-1-x_{20}-x_{12} x_{10}-4 h \sigma(1-\sigma)^{-1}\left(x_{10} \ln x_{10}\right), \\
& v_{02}(r) \equiv v_{0}\left(\mathbf{h}^{* *}\right)-v_{2}\left(\mathbf{h}^{* *}\right)=-(4 h+1)\left(x_{20}\right)^{2}+2(1+2 h) x_{20}-1-4 h \sigma(1-\sigma)^{-1}\left(x_{20} \ln x_{20}\right) .
\end{aligned}
$$

Differentiating $v_{0 i}(r)$ with respect to $r$, we have

$$
\begin{aligned}
& \frac{d v_{0 i}(r)}{d r}=\frac{d x_{i 0}}{d r} \alpha_{0 i}(r) \\
& \alpha_{01}(r) \equiv-2(1+4 h) x_{10}-\frac{4 h \sigma}{\sigma-1} \ln x_{10}-\beta(r)+4-\frac{4 h}{\sigma-1} \\
& \alpha_{02}(r) \equiv-2(1+4 h) x_{20}-\frac{4 h \sigma}{\sigma-1} \ln x_{20}+4-\frac{4 h}{\sigma-1} \\
& \beta(r) \equiv \frac{d\left(x_{20}+x_{12} x_{10}\right) / d r}{d x_{10} / d r}=\frac{6 r\left(1-r^{4}\right)}{1+3 r^{2}-3 r^{4}-r^{6}} \\
& \frac{d x_{10}}{d r}=\frac{\left(1-r^{6}\right)+3 r^{2}\left(1-r^{2}\right)}{\left(1+r^{4}\right)^{2}}>0, \quad \frac{d x_{20}}{d r}=\frac{4 r\left(1-r^{4}\right)}{\left(1+r^{4}\right)^{2}}>0
\end{aligned}
$$

From the fact that $\alpha_{0 i}(r)$ is a monotonically decreasing function of $r, \alpha_{0 i}(0)>0$ and $\alpha_{0 i}(1)<0$, $v_{0 i}(r)$ is a unimodal function of $r$. Therefore, we can show that

$$
v_{0 i}(r)\left\{\begin{array}{l}
<0 \text { for } 0<r<r_{0 i}^{* *} \\
\geq 0 \text { for } r_{0 i}^{* *} \leq r<1
\end{array} \quad(i=1,2), \text { and } 0<r_{01}^{* *}<r_{02}^{* *},\right.
$$

where $r_{0 i}^{* *}$ is the solution of $v_{0 i}(r)=0 \quad(i=1,2)$. That is, the equilibrium condition for $\mathbf{h}^{* *}$, $v_{0}\left(\mathbf{h}^{* *}\right)=\max _{k}\left\{v_{k}\left(\mathbf{h}^{* *}\right)\right\}$, is satisfied for any $r$ larger than $r_{02}^{* *}$, and $r=r_{02}^{* *}$ is the sustain point for $\mathbf{h}^{* *}$.

\section{Appendix 4: Proof of Lemmas 6.1.1 and 6.1.2}

Proof of Lemma 6.1.1: We prove that each of the submatrices $\mathbf{J}^{(i j)}$ and $\mathbf{V}^{(i j)}$ is a circulant in turn. 
(1) Let each of $p_{(0)}$ and $p_{(1)}$ denotes the location choice probability of the subset of cities $C_{0}(i=0)$ and $C_{1}(i=1)$ at the agglomeration pattern $\mathbf{h}^{*}$, respectively:

$$
p_{(i)} \equiv \frac{\exp \left[-\theta v_{i}\left(\mathbf{h}^{*}\right)\right]}{M\left\{\exp \left[-\theta v_{0}\left(\mathbf{h}^{*}\right)\right]+\exp \left[-\theta v_{1}\left(\mathbf{h}^{*}\right)\right]\right\}}=\left\{\begin{array}{ll}
(1-\varepsilon) / M & (i=0) \\
\varepsilon / M & (i=1)
\end{array} .\right.
$$

A straightforward calculation of the definition (6.6a) of $\mathbf{J}^{\times}\left(\mathbf{h}^{*}\right) \equiv \mathbf{P}_{(1)} \mathbf{J}\left(\mathbf{h}^{*}\right) \mathbf{P}_{(1)}^{\mathrm{T}}$ reveals that the submatrices $\mathbf{J}^{(i j)}(i, j=0,1)$ are circulants whose first row vector is given by

$$
\mathbf{J}_{0}^{(i j)}=\left\{\begin{array}{l}
\theta p_{(i)}\left[1-p_{(j)},-p_{(j)}, \ldots,-p_{(j)}\right](i=j) \\
\theta p_{(i)}\left[-p_{(j)},-p_{(j)}, \ldots,-p_{(j)}\right] \quad(i \neq j)
\end{array} .\right.
$$

(2) As is shown in (3.19), the Jacobian matrix $\nabla \mathbf{v}\left(\mathbf{h}^{*}\right)$ consists of additions and multiplications of $\mathbf{M}\left(\mathbf{h}^{*}\right) \equiv \mathbf{D}\left\{\boldsymbol{\Delta}\left(\mathbf{h}^{*}\right)\right\}^{-1}$. It follows from this that the Jacobian matrix $\nabla^{\times} \mathbf{v}\left(\mathbf{h}^{*}\right) \equiv \mathbf{P}_{(1)} \nabla \mathbf{v}\left(\mathbf{h}^{*}\right) \mathbf{P}_{(1)}^{\mathrm{T}}$ defined in (6.6b) consists of those of $\mathbf{P}_{(1)} \mathbf{M}\left(\mathbf{h}^{*}\right) \mathbf{P}_{(1)}^{\mathrm{T}}$, which in turn is composed of submatrices $\mathbf{M}^{(i j)}(i, j=0,1)$ :

$$
\mathbf{P}_{(1)} \mathbf{M}\left(\mathbf{h}^{*}\right) \mathbf{P}_{(1)}^{\mathrm{T}} \equiv \frac{1}{2 h}\left[\begin{array}{c:c}
\mathbf{M}^{(00)} & \mathbf{M}^{(01)} \\
\hdashline \mathbf{M}^{(10)} & \mathbf{M}^{(11)}
\end{array}\right] .
$$

Therefore, in order to prove that $\mathbf{V}^{(i j)}(i, j=0,1)$ are circulants, it suffices to show that the submatrices $\mathbf{M}^{(i j)}(i, j=0,1)$ are circulants. Note here that $\mathbf{P}_{(1)} \mathbf{M}\left(\mathbf{h}^{*}\right) \mathbf{P}_{(1)}^{\mathrm{T}}$ can be represented as

$$
\mathbf{P}_{(1)} \mathbf{M}\left(\mathbf{h}^{*}\right) \mathbf{P}_{(1)}^{\mathrm{T}}=\left[\mathbf{P}_{(1)} \mathbf{D} \mathbf{P}_{(1)}^{\mathrm{T}}\right]\left[\mathbf{P}_{(1)}\left\{\boldsymbol{\Delta}\left(\mathbf{h}^{*}\right)\right\}^{-1} \mathbf{P}_{(1)}^{\mathrm{T}}\right] .
$$

The first bracket of the right-hand side of (A4.2) is given in (6.4), and a simple calculation of the second bracket yields

$$
\mathbf{P}_{(1)}\left\{\boldsymbol{\Delta}\left(\mathbf{h}^{*}\right)\right\}^{-1} \mathbf{P}_{(1)}^{\mathrm{T}}=\frac{1}{2 h}\left[\begin{array}{c:c}
\operatorname{diag}\left[d_{(0)}, \ldots, d_{(0)}\right] & \mathbf{0} \\
\hdashline \mathbf{0} & \operatorname{diag}\left[d_{(1)}, \ldots, d_{(1)}\right]
\end{array}\right]^{-1},
$$

where $d_{(0)} \equiv \mathbf{d}_{0}^{(0)} \cdot \mathbf{1}=\frac{1+r^{2}}{1-r^{2}}\left(1-r^{M}\right)$ and $d_{(1)} \equiv \mathbf{d}_{0}^{(1)} \cdot \mathbf{1}=\frac{2 r}{1-r^{2}}\left(1-r^{M}\right)$. Thus, we have

$$
\mathbf{P}_{(1)} \mathbf{M}\left(\mathbf{h}^{*}\right) \mathbf{P}_{(1)}^{\mathrm{T}}=\frac{1}{2 h}\left[\begin{array}{c:c:c}
d_{(0)}^{-1} \mathbf{D}_{(0)} & d_{(0)}^{-1} \mathbf{D}_{(1)} \\
\hdashline d_{(1)}^{-1} \mathbf{D}_{(1)} & d_{(1)}^{-1} \mathbf{D}_{(0)}
\end{array}\right],
$$

which shows that the submatrices $\mathbf{M}^{(i j)}(i, j=0,1)$ are circulants. QED

Proof of Lemma 6.1.2: Consider the Jacobian matrix $\nabla^{\times} \mathbf{F}\left(\mathbf{h}^{*}\right)$ defined in (6.5):

$$
\begin{aligned}
& \nabla^{\times} \mathbf{F}\left(\mathbf{h}^{*}\right) \equiv \mathbf{P} \nabla \mathbf{F}\left(\mathbf{h}^{*}\right) \mathbf{P}^{\mathrm{T}}=H \mathbf{J}^{\times}\left(\mathbf{h}^{*}\right) \nabla^{\times} \mathbf{v}\left(\mathbf{h}^{*}\right)-\mathbf{I} \equiv M\left[\begin{array}{c:c}
\mathbf{F}^{(00)} & \mathbf{F}^{(01)} \\
\hdashline \mathbf{F}^{(10)} & \mathbf{F}^{(11)}
\end{array}\right]-\mathbf{I}, \\
& \mathbf{F}^{(i j)} \equiv \sum_{k=0,1} \mathbf{J}^{(i k)} \mathbf{V}^{(k j)} \quad(i, j=0,1) .
\end{aligned}
$$

Note here that the submatrices $\mathbf{F}^{(i j)}$ of the Jacobian matrix $\nabla^{\times} \mathbf{F}\left(\mathbf{h}^{*}\right)$ are circulants because all submatrices $\mathbf{J}^{(i j)}$ and $\mathbf{V}^{(i j)}(i, j=0,1)$ are circulants. This enables us to diagonalize each of the 
submatrices $\mathbf{F}^{(i j)}$ by using a $M$ by $M$ DFT matrix $\mathbf{Z}_{[M]}$ :

$$
\left(\mathbf{Z}^{\times}\right)^{-1} \nabla^{\times} \mathbf{F} \mathbf{Z}^{\times}=M\left[\begin{array}{c:c}
\operatorname{diag}\left[\mathbf{f}^{(00)}\right] & \operatorname{diag}\left[\mathbf{f}^{(01)}\right] \\
\hdashline \operatorname{diag}\left[\mathbf{f}^{(10)}\right] & \operatorname{diag}\left[\mathbf{f}^{(11)}\right]
\end{array}\right]-\mathbf{I},
$$

where $\mathbf{f}^{(i j)}$ is the eigenvalues of $\mathbf{F}^{(i j)}$, and $\mathbf{Z}^{\times} \equiv \operatorname{diag}\left[\mathbf{Z}_{[M]}, \mathbf{Z}_{[M]}\right]$. It also follows that applying the similarity transformation based on $\mathbf{Z}_{[M]}$ to both sides of (A4.4b) yields

$$
\mathbf{f}^{(i j)}=\sum_{k=0,1}\left[\boldsymbol{\delta}^{(i k)}\right] \cdot\left[\mathbf{e}^{(k j)}\right] \quad(i, j=0,1),
$$

where $\delta^{(i j)} \equiv\left[\delta_{0}^{(i j)}, \delta_{1}^{(i j)}, \ldots, \delta_{M-1}^{(i j)}\right]^{\mathrm{T}}$ and $\mathbf{e}^{(i j)} \equiv\left[e_{0}^{(i j)}, e_{1}^{(i j)}, \ldots, e_{M-1}^{(i j)}\right]^{\mathrm{T}}$ denote the eigenvalues of the Jacobian matrices $\mathbf{J}^{(i j)}$ and $\mathbf{V}^{(i j)}(i, j=0,1)$, respectively. The former eigenvalues $\delta^{(i j)}$ can be given analytically by the DFT of the first row vector $\mathbf{J}_{0}{ }^{(i j)}$ of the submatrix $\mathbf{J}^{(i j)}$ :

$$
\delta^{(i j)}=\mathbf{Z}_{[M]} \mathbf{J}_{0}^{(i j)} \quad(i, j=0,1),
$$

Substituting (A4.1) into (A4.7), we have

$$
\begin{aligned}
& \delta^{(00)}=(\theta / M)(1-\varepsilon)\left[\begin{array}{llll}
\varepsilon & 1 & \cdots & 1
\end{array}\right]^{\mathrm{T}}, \quad \delta^{(11)}=(\theta / M) \varepsilon\left[\begin{array}{lllll}
1-\varepsilon & 1 & \cdots & 1
\end{array}\right]^{\mathrm{T}}, \\
& \delta^{(01)}=(\theta / M)(1-\varepsilon)\left[\begin{array}{llll}
-\varepsilon & 0 & \cdots & 0
\end{array}\right]^{\mathrm{T}}, \boldsymbol{\delta}^{(10)}=(\theta / M) \varepsilon\left[\begin{array}{llll}
\varepsilon-1 & 0 & \cdots & 0
\end{array}\right]^{\mathrm{T}} .
\end{aligned}
$$

It follows from this that

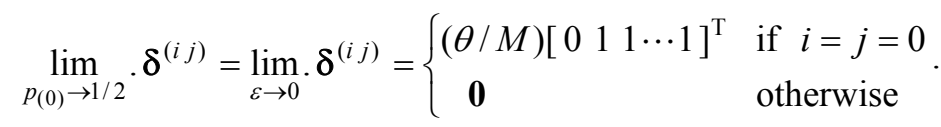

Substituting these $\delta^{(i j)}(i, j=0,1)$ into (A4.6) yields

$$
\lim _{p_{(0)} \rightarrow 1 / 2} .^{(i j)}=\left\{\begin{array}{ll}
(\theta / M)\left[\begin{array}{ll}
0 & e_{1}^{(0 j)} e_{2}^{(0 j)} \cdots e_{M-1}^{(0 j)}
\end{array}\right]^{\mathrm{T}} & \text { if } i=0 \\
\mathbf{0} & \text { if } i=1
\end{array} \quad(j=0,1) .\right.
$$

Thus, when $p_{(0)} \rightarrow 1 / 2$, (A4.5) reduces to

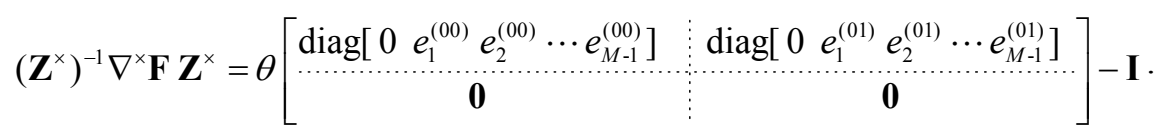

Converting this into the original coordinate system, we obtain

$$
\mathbf{P}^{\mathrm{T}}\left[\left(\mathbf{Z}^{\times}\right)^{-1} \nabla^{\times} \mathbf{F} \mathbf{Z}^{\times}\right] \mathbf{P}=\left(\mathbf{Z}^{*}\right)^{-1} \nabla \mathbf{F} \mathbf{Z}^{*}=\theta \operatorname{diag}\left[\hat{\mathbf{e}}_{0} \quad \hat{\mathbf{e}}_{1} \quad \cdots \quad \hat{\mathbf{e}}_{M-1}\right]-\mathbf{I}
$$

where $\mathbf{Z}^{*} \equiv \mathbf{P}^{\mathrm{T}} \mathbf{Z}^{\times} \mathbf{P} ; \hat{\mathbf{e}}_{0} \equiv\left[\begin{array}{ll}0 & 0 \\ 0 & 0\end{array}\right]$ and $\hat{\mathbf{e}}_{k} \equiv\left[\begin{array}{cc}e_{k}^{(00)} & e_{k}^{(01)} \\ 0 & 0\end{array}\right] \quad(k=1,2, \ldots, M-1)$.

Since eigenvalues of an upper-triangular matrix $\left(\hat{\mathbf{e}}_{k}\right)$ are given by the diagonal entries, we can conclude that the eigenvalues of the Jacobian $\nabla \mathbf{F}\left(\mathbf{h}^{*}\right)$ are given by

$$
g_{k}=\left\{\begin{array}{lc}
\theta e_{k / 2}^{(00)}-1 & (k: \text { even }) \\
-1 & (k: \text { odd })
\end{array} .\right.
$$

\section{Appendix 5: Proof of Lemma 6.1.3 and Proposition 6.1}

Proof of Lemma 6.1.3: Substituting (3.19) and (A4.3) into the definition of $\nabla^{\times} \mathbf{v}\left(\mathbf{h}^{*}\right)$ in (6.6), we 
obtain (6.8a) in lemma 6.1.3. Since the right-hand side of (6.8a) consisting only of circulants $\mathbf{D}^{(0)}$, $\mathbf{D}^{(1)}$ and $\mathbf{D}^{(1) \mathrm{T}}$, we have the associate eigenvalues $\mathbf{e}^{(00)}$ by $(6.8 b)$. QED

Proof of Proposition 6.1: Each of the eigenvalues $\mathbf{f}_{(i)}(i=0,1)$ of $\mathbf{D}^{(i)} / d_{(i)}$ is obtained by DFT of vectors $\mathbf{d}_{0}^{(i)}$ :

$$
\mathbf{f}_{(0)}=\mathbf{Z}_{[M]} \mathbf{d}_{0}^{(0)} / d_{(0)}, \quad \mathbf{f}_{(1)}=\mathbf{Z}_{[M]} \mathbf{d}_{0}^{(1)} / d_{(1)}, \quad \mathbf{f}_{(1 \mathrm{~T})}=\mathbf{Z}_{[M]} \mathbf{d}_{0}^{(1 \mathrm{~T})} / d_{(1)} .
$$

A straight-forward calculation of (A5.1) yields that

$$
f_{M / 2}^{(0)}=c\left(r^{2}\right)^{2}, \quad f_{M / 2}^{(1)}=f_{M / 2}^{(1 \mathrm{~T})}=0, \quad f_{m}^{(1)} f_{m}^{(1 \mathrm{~T})}>0 \quad(m=0,1,2, \ldots, M-1) .
$$

Substituting these into $(6.8 \mathrm{~b})$ yields

$$
\begin{array}{lrl}
e_{0}^{(00)}=G^{(00)}(1,1)=\text { const. }, & e_{M / 2}^{(00)}=G^{(00)}\left(c\left(r^{2}\right)^{2}, 0\right), \\
e_{m}^{(00)}=e_{M-m}^{(00)}=G^{(00)}\left(f_{m}^{(0)}, f_{m}^{(1)} f_{m}^{(1 \mathrm{~T})}\right) & (m=0,1,2, \ldots, M-1),
\end{array}
$$

where $G^{(00)}(x, y) \equiv b x-a_{1}\left[(1+2 h) x^{2}+y\right]$. It follows from (A5.3) that

$$
\max _{m} \cdot\left\{e_{m}^{*}(x)\right\}=G^{(00)}\left(c\left(r^{2}\right)^{2}, 0\right) \text {. }
$$

Combining this with (A4.8) in Lemma 6.1.2, we obtain (6.9a).QED

\section{Appendix 6: An Example of the division of the set $C$}

We show an example of the division of the set $C$ and the associated permutation $\sigma_{K}^{n}$ for the case of $K=16$. For an 8-centric configuration $\mathbf{h}^{*}$ that emerges after a first bifurcation from the flat earth equilibrium ( $n=1)$, the set $C$ with 16 elements is divided into 2 subsets, $C_{0}$ and $C_{1}$, each of which is a set of core regions and that of periphery regions, respectively. After a second bifurcation from the 8-centric configuration $\mathbf{h}^{*}(n=2)$, a 4-centric configuration $\mathbf{h}^{* *}$ emerges, and then each of the subsets $C_{i}(i=0,1)$ is divided into 2 subsets:

$$
\begin{array}{ll}
i=0: & C_{00}=\{0,4,8,12\} \quad \text { and } C_{01}=\{2,6,10,14\}, \\
i=1: & C_{10}=\{1,5,9,13\} \quad \text { and } C_{11}=\{3,7,11,15\},
\end{array}
$$

where $C_{00}$ is the set of core regions, and all other subsets are the sets of periphery regions in the 4centric configuration $\mathbf{h}^{* *}$. For a duo-centric configuration $\mathbf{h}^{(3)}$, which emerges after a third bifurcation $(n=3)$, each of the subsets $\left\{C_{i_{0} i_{1}}\left(i_{0}, i_{1}=0,1\right)\right\}$ is further divided into 2 subsets:

$$
\begin{aligned}
& i_{0} i_{1}=00: C_{000}=\{0,8\} \text { and } C_{001}=\{4,12\}, i_{0} i_{1}=01: C_{010}=\{2,10\} \text { and } C_{011}=\{6,14\}, \\
& i_{0} i_{1}=10: C_{100}=\{1,9\} \text { and } C_{101}=\{5,13\}, i_{0} i_{1}=11: C_{110}=\{3,11\} \text { and } C_{111}=\{7,15\} .
\end{aligned}
$$

where only the two regions in the subset $C_{000}$ has skilled workers. Thus, after the third bifurcation, $C$ is divided into $2^{3}=8$ subsets $\left\{C_{i_{0} i_{1} i_{2}}\left(i_{0}, i_{1}, i_{2}=0,1\right)\right\}$. Accordingly, the permutation $\sigma_{16}^{3}$ corresponding to this division of $C$ is given by

$$
\sigma_{16}^{3}:\left[\begin{array}{rr:rr:rr:rr:rr:rr:rr:rr}
0 & 1 & 2 & 3 & 4 & 5 & 6 & 7 & 8 & 9 & 10 & 11 & 12 & 13 & 14 & 15 \\
0 & 8 & 4 & 12 & 2 & 10 & 6 & 14 & 1 & 9 & 5 & 13 & 3 & 11 & 7 & 15
\end{array}\right] .
$$

It is readily verified that $\sigma_{K}^{n}(k)$ is given by the bit-reversal operation; for example, $k=12$ is written as "1100" $(\bmod 2)$, whose bit-reversal yields "0011" ( $\bmod 2)$, and hence, $\sigma_{16}^{3}(12)=0011(\bmod 2)=3$. This also shows that the first component of $C_{110}$ is given by the bit 
reversal of “110" $(\bmod 2)$.

\section{Appendix 7: Proof of Lemmas 6.2.1 and 6.2.2}

Proof of Lemma 6.2.1: We prove that the submatrices $\mathbf{J}^{(0, a(n))}$ and $\mathbf{V}^{(0, a(n))}$ are circulants in turn. (1) Let $p_{(a(n))}$ denotes the location choice probability of the subset of cities $C_{i_{0} i_{1} i_{2} \ldots i_{n-1}}$ at $\mathbf{h}=\mathbf{h}^{(n)}$ :

$$
p_{(a(n))}=\frac{\exp \left[-\theta v_{a(n)}\right]}{K / 2^{n}\left\{\sum_{k} \exp \left[-\theta v_{k}\right]\right\}}=\left\{\begin{array}{ll}
2^{n} / K & \text { if } a(n)=0 . \\
0 & \text { otherwise }
\end{array} .\right.
$$

Substituting this into (6.18a), we have

$$
\mathbf{J}^{(i j)}=\left\{\begin{array}{ll}
\left(\theta 2^{n} / K\right)\left(\mathbf{I}-\mathbf{1} \mathbf{1}^{\mathrm{T}}\right) & \text { if } i=j=0 \\
\mathbf{0} & \text { otherwise }
\end{array} .\right.
$$

This shows that the submatrices $\mathbf{J}^{(0, a(n))}$ are circulants.

(2) Since $\nabla \mathbf{v}\left(\mathbf{h}^{(n)}\right)$ consists of additions and multiplications of $\mathbf{M}\left(\mathbf{h}^{(n)}\right) \equiv \mathbf{D}\left\{\boldsymbol{\Delta}\left(\mathbf{h}^{(n)}\right)\right\}^{-1}$, $\nabla^{\times} \mathbf{v}\left(\mathbf{h}^{(n)}\right)$ defined in (6.18b) also consists of those of $\mathbf{P}_{(n)} \mathbf{M}\left(\mathbf{h}^{(n)}\right) \mathbf{P}_{(n)}^{\mathrm{T}}$. From this, in order to prove that $\mathbf{V}^{(0, a(n))}$ are circulants, it is suffices to show that the submatrices $\mathbf{M}^{(i, j)}$ of $\mathbf{P}_{(n)} \mathbf{M}\left(\mathbf{h}^{(n)}\right) \mathbf{P}_{(n)}^{\mathrm{T}}$ are circulants. $\mathbf{P}_{(n)} \mathbf{M}\left(\mathbf{h}^{(n)}\right) \mathbf{P}_{(n)}^{\mathrm{T}}$ is expressed as

$$
\mathbf{P}_{(n)} \mathbf{M}\left(\mathbf{h}^{(n)}\right) \mathbf{P}_{(n)}^{\mathrm{T}}=\left[\mathbf{P}_{(n)} \mathbf{D} \mathbf{P}_{(n)}^{\mathrm{T}}\right]\left[\mathbf{P}_{(n)}\left\{\boldsymbol{\Delta}\left(\mathbf{h}^{(n)}\right)\right\}^{-1} \mathbf{P}_{(n)}^{\mathrm{T}}\right] .
$$

The submatrices $\Delta^{(i, j)}$ of the second bracket of (A7.2) is given by

$$
\boldsymbol{\Delta}^{(i, j)}= \begin{cases}\left(2^{n} h\right)^{-1} \operatorname{diag}\left[d_{(a(n))} \mathbf{1}\right] & \text { if } i=j=a(n), \\ \mathbf{0} & \text { otherwise }\end{cases}
$$

where $d_{(a(n))} \equiv \mathbf{d}_{0}^{(a(n))} \cdot \mathbf{1}=\frac{r^{a(n)}+r^{2^{n}-a(n)}}{1-r^{2^{n}}}\left(1-r^{K / 2}\right)$, and this shows that $\Delta^{(i, j)}$ are circulants. As is shown in Section 6.2, the submatrices of the first bracket of the right-hand side of (A7.2) defined in (6.15) are circulants. Thus, $\mathbf{M}^{(i, j)}$ are circulants. QED

Proof of Lemma 6.2.2: The Jacobian matrix $\nabla^{\times} \mathbf{F}\left(\mathbf{h}^{(n)}\right)$ defined in (6.17) is given by

$$
\nabla^{\times} \mathbf{F}\left(\mathbf{h}^{(n)}\right)=H \mathbf{J}^{\times}\left(\mathbf{h}^{(n)}\right) \nabla^{\times} \mathbf{v}\left(\mathbf{h}^{(n)}\right) \equiv L(n)\left[\begin{array}{c:c:c:c}
\mathbf{F}^{(0,0)} & \mathbf{F}^{(0,1)} & \ldots & \mathbf{F}^{\left(0,2^{n-1}\right)} \\
\hdashline \mathbf{F}^{(1,0)} & \mathbf{F}^{(1,1)} & \ldots & \mathbf{F}^{\left(1,2^{n-1}\right)} \\
\hdashline \vdots & \vdots & \vdots & \vdots \\
\hdashline \mathbf{F}^{\left(2^{n-1}, 0\right)} & \mathbf{F}^{\left(2^{n-1}, 1\right)} & \ldots & \left.\mathbf{F}^{\left(2^{n-1}, 2^{n-1}\right.}\right)
\end{array}\right]-\mathbf{I},
$$

where $\mathbf{F}^{(i, j)} \equiv \sum_{k} \mathbf{J}^{(i, k)} \mathbf{V}^{(k, j)}, L(n) \equiv K / 2^{n}$. Since $\mathbf{F}^{(i, j)}$ are circulants, these submatrices are diagonalized by $L(n)$-by- $L(n)$ DFT matrix $\mathbf{Z}_{[L(n)]}$ :

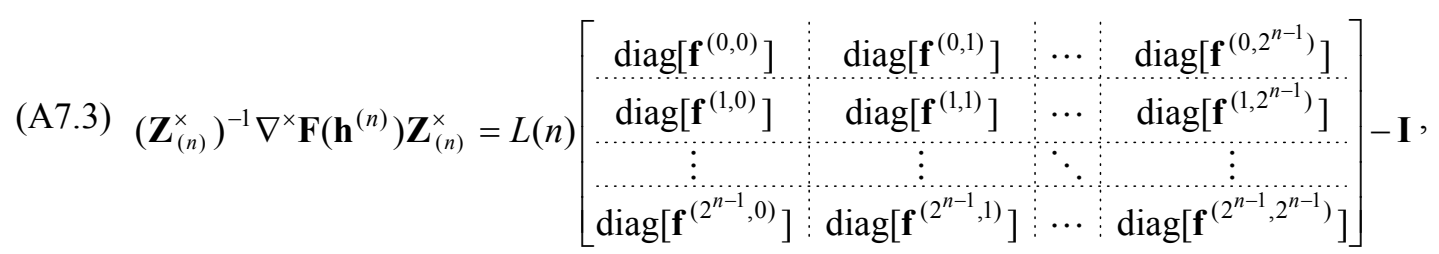


where $\mathbf{Z}_{(n)}^{\times} \equiv \operatorname{diag}\left[\mathbf{Z}_{[L(n)]}, \mathbf{Z}_{[L(n)]}, \ldots, \mathbf{Z}_{[L(n)]}\right]$, and $\mathbf{f}^{(i, j)}$ is the eigenvalues of $\mathbf{F}^{(i, j)}$, which is given by

$$
\mathbf{f}^{(i, j)}=\sum_{k}\left[\boldsymbol{\delta}^{(i, k)}\right] \cdot\left[\mathbf{e}^{(k, j)}\right]
$$

$\boldsymbol{\delta}^{(i, j)}=\left[\delta_{0}^{(i, j)}, \delta_{1}^{(i, j)}, \ldots, \delta_{L(n)}^{(i, j)}\right]^{\mathrm{T}}$ and $\mathbf{e}^{(i, j)}=\left[e_{0}^{(i, j)}, e_{1}^{(i, j)}, \ldots, e_{L(n)}^{(i, j)}\right]^{\mathrm{T}}$ denote the eigenvalues of $\mathbf{J}^{(i, j)}$ and $\mathbf{V}^{(i, j)}$, respectively. Applying the similarity transformation based on $\mathbf{Z}_{[L(n)]}$ to both sides of (A7.1), we have

$$
\boldsymbol{\delta}^{(i, j)}=\left\{\begin{array}{ll}
(\theta / L(n))[0,1,1, \cdots, 1]^{\mathrm{T}} & \text { if } i=j=0 \\
\mathbf{0} & \text { otherwise }
\end{array} .\right.
$$

Substituting this into (A7.4) yields

$$
\mathbf{f}^{(i, j)}=\left\{\begin{array}{ll}
(\theta / L(n))\left[0, e_{1}^{(0, j)}, e_{2}^{(0, j)}, \cdots, e_{L(n)}^{(0, j)}\right]^{\mathrm{T}} & \text { if } i=0 \\
\mathbf{0} & \text { otherwise }
\end{array} .\right.
$$

Thus, converting (A7.3) into the original coordinate system, we obtain

$$
\mathbf{P}_{(n)}^{\mathrm{T}}\left(\mathbf{Z}_{(n)}^{\times}\right)^{-1} \nabla^{\times} \mathbf{F}\left(\mathbf{h}^{(n)}\right) \mathbf{Z}_{(n)}^{\times} \mathbf{P}_{(n)}=\left(\mathbf{Z}_{(n)}^{*}\right)^{-1} \nabla^{\times} \mathbf{F}\left(\mathbf{h}^{(n)}\right) \mathbf{Z}_{(n)}^{*}=\theta \operatorname{diag}\left[\mathbf{0}, \hat{\mathbf{e}}_{1}^{(n)}, \ldots, \hat{\mathbf{e}}_{L(n)}^{(n)}\right]-\mathbf{I},
$$

where $\mathbf{Z}_{(n)}^{*} \equiv \mathbf{P}_{(n)}^{\mathrm{T}} \mathbf{Z}_{(n)}^{\times} \mathbf{P}_{(n)}$, and

$$
\hat{\mathbf{e}}_{k}^{(n)} \equiv\left[\begin{array}{cccc}
e_{k}^{(0,0)} & e_{k}^{(0,1)} & \cdots & e_{k}^{\left(0,2^{n-1}\right)} \\
0 & 0 & \cdots & 0 \\
\vdots & \vdots & \ddots & \vdots \\
0 & 0 & \cdots & 0
\end{array}\right] \quad(k=1,2, \ldots, L(n))
$$

Since eigenvalues of an upper-triangular matrix $\left(\hat{\mathbf{e}}_{k}^{(n)}\right)$ are given by the diagonal entries, we can conclude that the eigenvalues of the Jacobian are given by (6.19).

\section{Appendix 8: Proof of Lemma 6.2.3 and Proposition 6.3}

Proof of Lemma 6.2.3: Substituting (3.19) and (A7.2) into the definition of $\nabla^{\times} \mathbf{v}\left(\mathbf{h}^{(n)}\right)$ in (6.18b), we obtain (6.20a) in lemma 6.2.3. Since the right-hand side of (6.20a) consisting only of circulants $\mathbf{D}^{(0)}, \mathbf{D}^{(a(n))}$ and $\left\{\mathbf{D}^{(a(n))}\right\}^{\mathrm{T}}$, we have the associate eigenvalues $\mathbf{e}^{(00)}$ by $(6.20 \mathrm{~b})$. QED

Proof of Proposition 6.3: We see from Lemmas 6.2.2 and 6.2.3 that proposition 6.3 is equivalent to

(A8.1) $\quad \arg \cdot \max _{m} \cdot\left\{e_{m}^{(0,0)} \equiv E_{m}^{0}-E_{m}^{1}\right\}=M(n), \quad M(n) \equiv L(n) / 2 \equiv K / 2^{n+1}$,

$$
f_{M(n)}^{(0)} / d_{(0)}=c_{0}(r, n), \quad \sum_{a(n)=1}^{2^{n}-1} f_{M(n)}^{(a(n))} \cdot f_{M(n)}^{(a(n)) \mathrm{T}} / d_{(a(n))}^{2}=c_{0}(r, n) \sum_{j=1}^{2^{n}-1} c_{j}(r, n),
$$

where $\quad E_{m}^{0} \equiv\left(f_{m}^{(0)} / d_{(0)}\right)\left\{b-a_{n}\left(1+2^{n} h\right)\left(f_{m}^{(0)} / d_{(0)}\right)\right\}$,

$$
E_{m}^{1} \equiv a_{n} \sum_{a(n)=1}^{2^{n}-1} f_{m}^{(a(n))} \cdot f_{m}^{(a(n)) \mathrm{T}} / d_{(a(n))}^{2} \quad(m=0,1, \cdots, L(n)-1) .
$$


To prove these, we first derive analytical expressions of the eigenvalues $f_{m}^{(a(n))}$ and $f_{m}^{(a(n)) \mathrm{T}}$ $(m=0,1, \cdots, L(n)-1)$. Since $\mathbf{D}^{(a(n))}$ is a circulant, the eigenvalues $\left\{f_{m}^{(a(n))}\right\}$ are obtained by DFT of $\mathbf{d}_{0}^{(a(n))}: \mathbf{f}^{(a(n))}=\mathbf{Z}_{[L(n)]} \mathbf{d}_{0}^{(a(n))}$. Substituting (6.16) into this and some calculation yields

(A8.3a)

$$
f_{m}^{(a(n))}=\left[\alpha_{m}(n)+\mathrm{i} \beta_{m}(n)\right] \cdot \gamma_{m}(n),
$$

where $\quad \cos _{m} \equiv \cos \left(2 \pi(m / L(n)), \sin _{m} \equiv \sin \left(2 \pi(m / L(n)), R_{n} \equiv r^{2^{n}}, A_{n} \equiv r^{a(n)}\right.\right.$,

$$
\begin{aligned}
& \alpha_{m}(n) \equiv A_{n} \cdot\left(1-R_{n} \cos _{m}\right)-A_{n}{ }^{-1} \cdot\left(1-R_{n}^{-1} \cos _{m}\right) \cdot R_{n}{ }^{2}, \\
& \beta_{m}(n) \equiv\left(A_{n}-A_{n}{ }^{-1}\right)\left(R_{n} \sin _{m}\right), \quad \gamma_{m}(n) \equiv \frac{1-(-1)^{m} R_{n}{ }^{M(n)}}{1-2 R_{n} \cos _{m}+R_{n}{ }^{2}}>0,
\end{aligned}
$$

In a similar manner, we obtain the eigenvalues of $\mathbf{D}^{(a(n)) \mathrm{T}}$ as

$$
f_{m}^{(a(n)) \mathrm{T}}=\left[\alpha_{m}(n)-\mathrm{i} \beta_{m}(n)\right] \cdot \gamma_{m}(n) .
$$

From (A8.3a) and (A8.3b), we have

$$
f_{m}^{(a(n))} \cdot f_{m}^{(a(n)) \mathrm{T}}=\left[\alpha(n)+\beta(n) \cos _{m}\right] \cdot \gamma_{m}(n)^{2},
$$

where $\quad \alpha(n) \equiv\left(1+R_{n}^{2}\right)\left(A_{n}-R_{n} A_{n}^{-1}\right)^{2}+\left(1+R_{n}\right)^{2}\left(2 R_{n}\right) \geq 0$,

$$
\beta(n) \equiv 2 R_{n} A_{n}^{-1}\left(A_{n}^{2}-1\right)\left(R_{n}^{2}-A_{n}^{2}\right) \geq 0 \text {. }
$$

To prove (A8.1), it suffices to show that

$$
\arg \cdot \max _{m} \cdot\left\{E_{m}^{0}\right\}=M(n) \text { and arg. } \min _{m} \cdot\left\{E_{m}^{1}\right\}=M(n) .
$$

The former is true since it is readily verified from (A8.3) that $\arg . \min _{m} .\left\{f_{m}^{(0)}\right\}=M(n)$. The latter is also evident from the fact that $\arg \cdot \min _{m} \cdot\left\{f_{m}^{(a(n))} \cdot f_{m}^{(a(n)) \mathrm{T}}\right\}=M(n)\left(a(n)=1,2, \cdots, 2^{n}-1\right)$, which is obvious from (A8.4) since $\alpha(n) \geq 0, \beta(n) \geq 0, \gamma_{m}(n) \geq 0$. Thus, (A8.1) has been proved. (A8.2) can be easily proved by calculating $d_{(a(n))}$ and $f_{M(n)}^{(a(n))} \cdot f_{M(n)}^{(a(n)) T}$ :

$$
\begin{aligned}
& d_{(a(n))}=\mathbf{1} \cdot \mathbf{d}_{0}^{(a(n))}=\left(r^{a(n)}+r^{2^{n}-a(n)}\right) \frac{1-r^{K / 2}}{1-r^{2^{n}}}, \\
& f_{M(n)}^{(a(n))} \cdot f_{M(n)}^{(a(n)) \mathrm{T}}= \begin{cases}\left(r^{a(n)}-r^{2^{n}-a(n)}\right)^{2} \frac{\left(1-r^{K / 2}\right)^{2}}{\left(1+r^{2^{n}}\right)^{2}} & \text { for } M(n) \geq 2 \\
\left(r^{a(n)}-r^{2^{n}-a(n)}\right)^{2} & \text { for } M(n)=1\end{cases}
\end{aligned}
$$

Setting $a(n)=0$ in (A8.3) and (A8.5) yields the first equality of (A8.2). The second equality of (A8.2) also can be readily verified by substituting (A8.5) and (A8.6) into the LHS. QED.

\section{Appendix 9: Proof of Lemma 6.2.4}

(i) Defining $\varepsilon_{n}(r) \equiv C_{2^{n} / 2}(r)+\hat{\varepsilon}_{n}(r), \hat{\varepsilon}_{n}(r) \equiv \sum_{k=1}^{\left(2^{n} / 2\right)-1} C_{k}(r)$ and $C_{k}(r) \equiv\left(\frac{1-r^{2 k}}{1+r^{2 k}}\right)^{2}$, we have

$$
\hat{G}^{(n)}(r)=-\sigma^{-1}\left\{c_{0}(r)+\left(2^{n} h\right)^{-1} \varepsilon_{n}(r)\right\}
$$


Since $d c_{0}(r, n) / d r<0$ and $d \varepsilon_{n}(r) / d r<0 \forall r \in(0,1]$, we can conclude that the first derivative of the RHS of (A9.1) is negative, that is, $d \hat{G}^{(n)}(r) / d r<0 \forall r \in(0,1]$.

(ii) Substituting $C_{k}(0)=1$ and $\varepsilon_{n}(0)=1+2\left\{\left(2^{n} / 2\right)-1\right\}=2^{n}-1$ into (A9.1), we obtain (6.32).

Substituting $C_{k}(1)=0$ and $\varepsilon_{n}(1)=0$ into (A9.1) yields $\hat{G}^{(n)}(1)=b>0$.

(iii) It follows from (A9.1) that

$$
\hat{G}^{(n)}(r)-\hat{G}^{(n+1)}(r)=\sigma^{-1}\left[\left\{c_{0}(r, n+1)-c_{0}(r, n)\right\}+\left(2^{n+1} h\right)^{-1}\left\{\varepsilon_{n+1}(r)-2 \varepsilon_{n}(r)\right\}\right] .
$$

To prove $\hat{G}^{(n)}(r)-\hat{G}^{(n+1)}(r) \geq 0$, it suffice to show that $\varepsilon_{n+1}-2 \varepsilon_{n} \geq 0$ since it is obvious that $c_{0}(r, n+1) \geq c_{0}(r, n)$ (the equality holds only if $\left.r=1\right)$. From the definition of $\varepsilon_{n}(r)$,

$$
\varepsilon_{n+1}-2 \varepsilon_{n}=C_{2^{n+1 / 2}}-2 C_{2^{n+1 / 2}}+2\left(\hat{\varepsilon}_{n+1}-2 \hat{\varepsilon}_{n}\right) .
$$

It also follows from the definitions of $\hat{\varepsilon}_{n}(r)$ and $C_{k}(r)$ that

$$
\begin{aligned}
\hat{\varepsilon}_{n+1}-2 \hat{\varepsilon}_{n}=\left(\hat{\varepsilon}_{n+1}-\hat{\varepsilon}_{n}\right)-\hat{\varepsilon}_{n} & =\sum_{k=2^{n} / 2}^{\left(2^{n+1} / 2\right)-1} C_{k}-\sum_{k=1}^{\left(2^{n} / 2\right)-1} C_{k} \\
& =C_{2^{n} / 2}+\sum_{k=1}^{\left(2^{n} / 2\right)-1}\left(C_{k+\left(2^{n} / 2\right)}-C_{k}\right) .
\end{aligned}
$$

Substituting (A9.4) into (A9.3), we have

$$
\varepsilon_{n+1}-2 \varepsilon_{n}=C_{2^{n+1 / 2}}+2 \sum_{k=1}^{\left(2^{n} / 2\right)-1}\left(C_{k+\left(2^{n} / 2\right)}-C_{k}\right) .
$$

Since $C_{k+\ell}(r) \geq C_{k}(r) \quad \forall \ell, k>0$, for any $0 \leq r \leq 1, n \geq 1$, we conclude that $\varepsilon_{n+1}-2 \varepsilon_{n} \geq 0$

(the equality holds only if $r=1$ ). QED.

\section{REFERENCES}

Akamatsu, T. and Takayama, Y. (2009a): “A Simplified Approach to Analyzing Multi-regional CorePeriphery Models," TUTUP Working Paper, Tohoku University.

Akamatsu, T. and Takayama, Y. (2009b): "Dispersion Forces Matter for the Emergence of Polycentric Urban Configurations," TUTUP Working Paper, Tohoku University.

Akamatsu, T., Takayama, Y. and Sugasawa, A. (2009): "Market Territories and Agglomeration Patterns in a Core-Periphery System of Cities Model," TUTUP Working Paper, Tohoku University.

Anas, A. (1983): "Discrete Choice Theory, Information Theory, and the Multinomial Logit and Gravity Models," Transportation Research B, 17, 13-23.

Anderson, J. (1979): “A Theoretical Foundation for the Gravity Equation," American Economic Review, 69, 106-116.

Anderson, J. and van Wincoop, E. (2003): "Gravity with Gravitas, A Solution to the Border Puzzle," American Economic Review, 93, 170-192.

Anderson, S.P., de Palma, A. and Thisse, J.-F. (1992): Discrete Choice Theory of Product Differentiation, MIT Press.

Baldwin, R., Forslid, R., Martin, P., Ottaviano, G. and Robert-Nicoud, F. (2003): Economic Geography and Public Policy, Princeton University Press.

Behrens, K. and Thisse, J.-F. (2007): "Regional Economics, A New Economic Geography Perspective," Regional Science and Urban Economics, 37, 457-465.

Bergstrand, J.H. (1989): "The Generalized Gravity Equation, Monopolistic Competition, and the 
Factor-Proportions Theory in International Trade," Review of Economics and Statistics, 71, 143153.

Berliant, M., Peng, S.K. and Wang, P. (2002): "Production Externalities and Urban Configuration," Journal of Economic Theory, 104, 275-303.

Berliant, M. and Wang, P. (2008): "Urban Growth and Subcenter Formation: A Trolley Ride from the Staples Center to Disneyland and the Rose Bowl," Journal of Urban Economics, 63, 679-693.

Christaller, W. (1933): Central Places in Southern Germany, In Charles W. Baskin (trans.) Die Zentralen Orte in Süddeutschland. Englewood Cliffs, NJ: Prentice Hall.

Combes, P-P., Mayer, T. and Thisse, J-F. (2008): Economic Geography: The Integration of Regions and Nations, Princeton University Press.

Dixit, A. and Stiglitz, J. (1977): "Monopolistic Competition and Optimum Product Diversity," American Economic Review, 67, 297-308.

Eaton, B.C. and Wooders, M.H. (1985): "Sophisticated Entry in a Model of Spatial Competition," RAND Journal of Economics, 16, 282-297.

Evenett, S. and Keller, W. (2002): "On Theories Explaining the Success of the Gravity Equation," Journal of Political Economy, 110, 281-316.

Flam, H. and Helpman, E. (1987): "Industrial Policy under Monopolistic Competition,” Journal of International Economics, 22, 79-102.

Forslid, R. and Ottaviano, G. (2003): “An Analytically Solvable Core -Periphery Model,” Journal of Economic Geography, 3, 229-240.

Fudenberg, D. and Levine, D. K., (1998): The Theory of Learning in Games, MIT Press.

Fujita, M. (1988): “A Monopolistic Competition Model of Spatial Agglomeration: Differentiated Product Approach," Regional Science and Urban Economics, 18, 87-124.

Fujita, M. and Mori, T., (1997): "Structural Stability and Evolution of Urban Systems," Regional Science and Urban Economics, 27, 399-442.

Fujita, M. Krugman, P., and Venables, A.J. (1999): The Spatial Economy, MIT Press.

Fujita, M. and Ogawa, H. (1982): Multiple Equilibria and Structural Transition of Non-monocentric Urban Configurations, Regional Science and Urban Economics, 12, 161-196.

Fujita, M. and Thisse, J.-F. (2002): Economics of Agglomeration, Cambridge University Press.

Fujita, M. and Thisse, J.-F. (2009): "New Economic Geography: An Appraisal on the Occasion of Paul Krugman's 2008 Nobel Prize in Economic Sciences," Regional Science and Urban Economics, 39, 109-119.

Glaeser, E.L. (2008): Cities, Agglomeration and Spatial Equilibrium, Oxford University Press.

Golubitsky, M. and Schaeffer, D.G. (1985): Singularities and Groups in Bifurcation Theory, Springer.

Gray, R. M. (2006): "Toeplitz and Circulant Matrices: A Review," Foundations and Trends in Communi-cations and Information Theory, 2, 155-239.

Hale, J. and Kocak, H. (1991): Dynamics and Bifurcations, Springer.

Helpman, E. (1998): "The Size of Regions," in Pines, D., Sadka, E. and Zilcha Y. (eds.), Topics in Public Economics. Theoretical and Applied Analysis, Cambridge University Press, 33-54.

Henderson, J.V. and Thisse, J.-F.(eds.) (2004): Handbook of Regional \& Urban Economics, 4, Elsevier.

Hirsch, M.W. and Smale, S. (1974): Differential Equations, Dynamical Systems, and Linear Algebra, Academic Press.

Hofbauer, J. and Sandholm, W. H. (2002): "On the Global Convergence of Stochastic Fictitious Play," Econometrica, 70, 2265-2294.

Hofbauer, J. and Sandholm, W. H. (2007): "Evolution in Games with Randomly Disturbed Payoffs," Journal of Economic Theory, 132, 47-69.

Ikeda, K., Akamatsu, T. and Kono, T. (2009): "Spatial Agglomeration Pattern of a System of Cities: Bifurcation Theory for Core-Periphery Model," TUTUP Working Paper, Tohoku University.

Krugman, P. (1991): “Increasing Returns and Economic Geography," Journal of Political Economy, 99, 483-499.

Krugman, P. (1993): "On the Number and Location of Cities," European Economic Review, 37, 293298.

Krugman, P. (1996): The Self-Organizing Economy, Blackwell Publishers.

Lucas, R.E. and Rossi-Hansberg, E. (2002): “On the Internal Structure of Cities,” Econometrica, 77, 
1445-1476.

Martin, P.J. and Rogers, C.A. (1995): “Trade Effects of Regional Aid,” in Baldwin, R., Haaparanta, P. and Kiander, J. (eds.), Expanding Membership of the European Union, Cambridge University Press, 166-188.

McFadden, D. (1974): “Conditional Logit Analysis of Qualitative Choice Behavior,” in Zarembka, P. (eds.), Frontiers in Econometrics, Academic Press, 105-142.

Mossay, P. (2003): "Increasing Returns and Heterogeneity in a Spatial Economy," Regional Science and Urban Economics, 33, 419-444.

Murata, Y. (2003): "Product Diversity, Taste Heterogeneity, and Geographic Distribution of Economic Activities: Market vs. Non-market Interactions," Journal of Urban Economics, 53, 126-144.

Novshek, W. (1980): "Equilibrium in Simple Spatial (or Differentiated Product) Models," Journal of Economic Theory, 22, 313-326.

Ottaviano, G., Tabuchi, T. and Thisse, J.-F. (2002): "Agglomeration and Trade Revisited," International Economic Review, 43, 409-436.

Oyama, D. (2009). "Agglomeration under Forward-looking Expectations: Potentials and Global Stability," Regional Science and Urban Economics, 39, 696-713.

Papageorgiou, Y.Y. and Smith, T.R. (1983): “Agglomeration as Local Instability of Spatially Uniform Steady-States," Econometrica, 51, 1109-1120.

Picard, P.M. and Tabuchi, T. (2009): "Self-Organized Agglomerations and Transport Costs," Economic Theory, 42, 565-589.

Pflüger, M. (2004): “A Simple Analytically Solvable, Chamberlinian Agglomeration Model,” Regional Science and Urban Economics, 34, 565-573.

Ravenstein, E.G. (1885): "The Laws of Migration," Journal of the Royal Statistical Society, 48, $167-$ 227.

Reilly, W.J. (1931): The Law of Retail Gravitation, Knickerbrocker Press.

Sandholm, W.H. (2009): Population Games and Evolutionary Dynamics, MIT Press.

Salop, S. (1979): "Monopolistic Competition with Outside Goods," Bell Journal of Economics, 10, 141-156.

Sen, A.K. and Smith, T.E. (1995): Gravity Models of Spatial Interaction Behavior, Springer.

Smith, T.E. (1976): "Spatial Discounting and the Gravity Hypothesis," Regional Science and Urban Economics, 6, 331-356.

Tabuchi, T. (1998): "Urban Agglomeration and Dispersion: A Synthesis of Alonso and Krugman," Journal of Urban Economics, 44, 333-351.

Tabuchi, T., Thisse, J.-F. and Zeng, D.Z. (2005): "On the Number and Size of Cities," Journal of Economic Geography, 5, 423-448.

Tabuchi, T., and Thisse, J.F. (2009): "Self-organizing Urban Hierarchy," CIRJE discussion paper, No. F-414, University of Tokyo.

Tinbergen, J. (1962): Shaping the World Economy: Suggestions for an International Economic Policy, Twentieth Century Fund.

Turing, A.M. (1952): "The Chemical Basis of Morphogenesis," Philosophical Transactions of the Royal Society of London, 237, 37-72.

Van Loan, C. (1992): Computational Frameworks for the Fast Fourier Transform, SIAM.

Voorhees, A.M. (1955): "A General Theory of Traffic Movement," Proceedings of the Institute of Traffic Engineers, 46-56.

Wilson, A.G. (1970): Entropy in Urban and Regional Modeling, Pion.

Young, E.C. (1924): “The Movement of Farm Population,” Cornell Agricultural Experimental Station, Bulletin 426.

Zipf, G.K. (1946): “The P1 P2/D Hypothesis: On the Intercity Movement of Persons," American Sociological Review, 11, 677-686. 